SCR-674 4 SP $^{17} 1965$

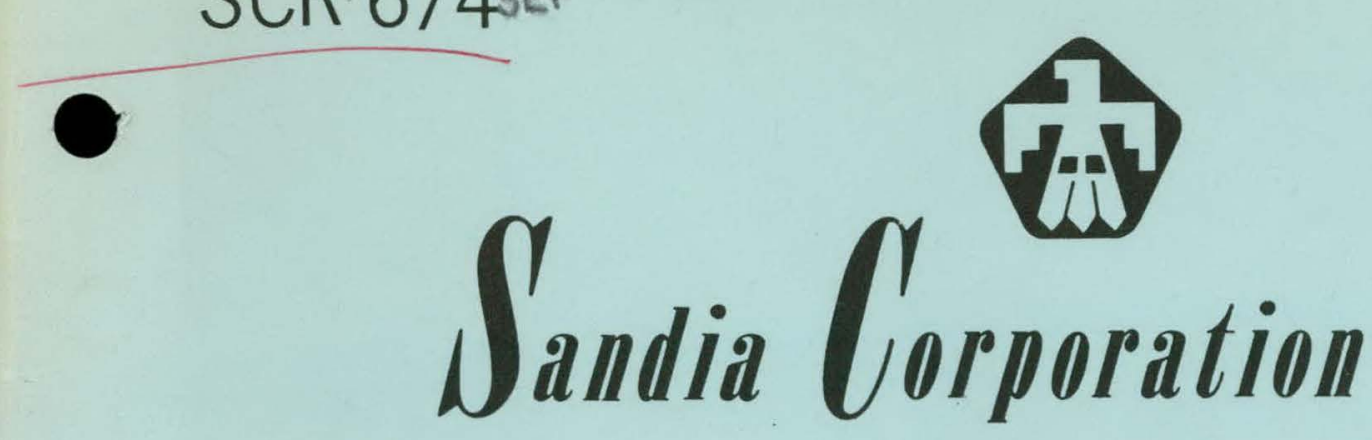

MASTER

$\bullet \bullet \bullet \bullet \bullet \bullet$ MONOGRAPH

\title{
THE ADMITTANCE OF BARE CIRCULAR LOOP ANTENNAS IN A DISSIPATIVE MEDIUM
}

\author{
by \\ R W P King, \\ C W Harrison, Jr \\ and \\ D G Tingley
}

Facsimile Price \$

Microfilm Price \$

Available from the

Office of Technical Services

Department of Commerce

Washington 25, D. C.

JUNE 1963 


\section{DISCLAIMER}

This report was prepared as an account of work sponsored by an agency of the United States Government. Neither the United States Government nor any agency Thereof, nor any of their employees, makes any warranty, express or implied, or assumes any legal liability or responsibility for the accuracy, completeness, or usefulness of any information, apparatus, product, or process disclosed, or represents that its use would not infringe privately owned rights. Reference herein to any specific commercial product, process, or service by trade name, trademark, manufacturer, or otherwise does not necessarily constitute or imply its endorsement, recommendation, or favoring by the United States Government or any agency thereof. The views and opinions of authors expressed herein do not necessarily state or reflect those of the United States Government or any agency thereof. 


\section{DISCLAIMER}

Portions of this document may be illegible in electronic image products. Images are produced from the best available original document. 


\section{Published by Sandia Corporation, a prime contractor to the \\ United States Atomic Energy Commission}

\section{LEGAL NOTICE}

This report was prepared as an account of Government sponsored work Neither the United States, nor the Commission, nor any person acting on behalf of the Commission:

A. Makes any warranty or representation, expressed or implied, with respect to the accuracy, completeness, or usefulness of the information contained in this report, or that the use of any information, apparatus, method, or process disclosed in this report may not infringe privately owned rights; or

B. Assumes any liabilities with respect to the use of, or for damages resulting from the use of any information, apparatus, method, or process disclosed in this report.

As used in the above, "person acting on behalf of the Commission" includes any employee or contractor of the Commission, or employee of such contractor to the extent that such employee or contractor of the Commission, or employee of such contractor prepares, disseminates, or provides access to, any information pursuant to his employment or contract with the Commission, or his employment with such contractor. 


\section{SANDIA CORPORATION MONOGRAPH}

'IHE ADMITTANCE OF BARE CIRCULAR LOOF ANTENNAS IN A DISSIPATIVE MEDIUM

R.W. P. King

Consultant to the Sandia Corporation and

Professor of Applied Physics, Harvard University

C.W. Harrison, Jr.

Member of the Technical Staff Sandia Corporation

D. G. Tingley

Gordon McKay Laboralory of Applied Science Cambridge, Massachusetts

June 1963 
The normalized input admittance of thin bare circular loop antennas has been evaluated from the theory of T. T. Wu. Computations have been made for loops in air and in an infinite homogeneous isotropic dissipative medium. A comparison is also made with Storer's theory of the loop. Numerical results are given in the form of graphs for several wire sizes and for loops up to two wavelengths in circumference. 'The properties of the medium are represented by the ratio $\alpha / \beta$ in the range from $z$ ero (perfect dielectric) to one (good conductor); $\alpha$ and $\beta$ are the imaginary and real parts of the complex propagation constant $\mathrm{k}=\beta-\mathrm{j} \alpha=\omega \sqrt{! !}(\epsilon-\mathrm{j \sigma} / \omega)$ where $\mu$ is the permeability, $\epsilon$ the dielectric constant and $\sigma$ the conductivity of the medium. 
Introduction

Analytical Formulation

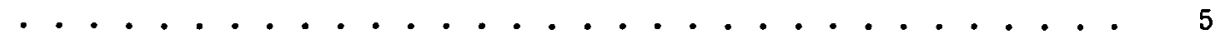

Evaluation of the Tables

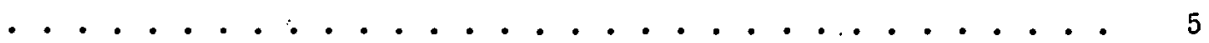

Graphical Representation

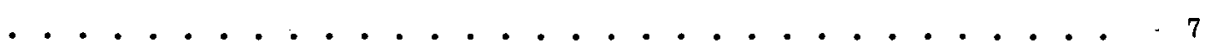

Conclusion

$$
\begin{aligned}
& \text {. }
\end{aligned}
$$

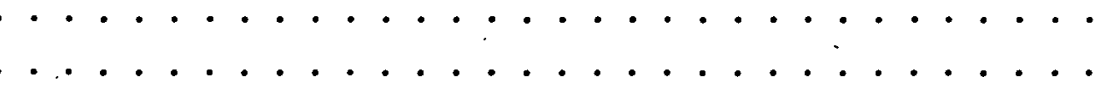

List of References

\section{LIS'I' UF' LLLUS'I'RATIUNS}

\section{Figure}

1 Normalized conductance and susceptance of circular loop in air as a function of the number of terms - Wu's theory for $\beta b=0.5$ and $2.0, \Omega=2 \ln (2 \pi b / a)=8-12 \ldots . .$.

2 Normalized susceptance of circular loop in a dissipative medium with propagation constant $\mathrm{k}=\beta-\mathrm{j} \alpha$ as a function of the number of terms - Wu's theory for $\beta \mathrm{b}=2.0, \Omega=8,12,20$, $\alpha / \beta=0-1 \ldots \ldots \ldots \ldots \ldots$

3 Normalized admittance of circular loop antenna in a dissipative medium - Wu's theory,

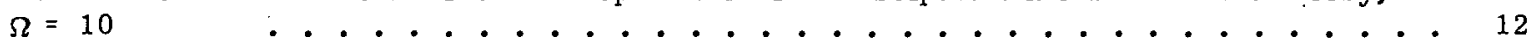

4 Normalized admittance of circular loop antenna in a dissipative medium - Wu's theory,

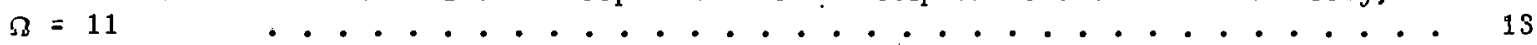

5 Normalized admittance of circular loop antenna in a dissipative medium - Wu's theory, $\Omega=12$

$$
\text { ............................... }
$$

6 Normalized admittance of circular loop antenna in a dissipative medium - Wu's theory, $\Omega=15$ .. . .............................

7 Normalized admittance of circular loop antenna in a dissipative medium - Wu's theory, $\Omega=17.5$

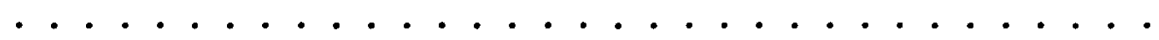

8 Normalized admittance of circular loop antenna in a dissipative medium - Wu's theory, $\Omega=20$

\section{LIST OF TABLES}

TABLE I Normalized Admittance $Y / \Delta$ of Loop Antennas in Dissipative Media . . . . 18-19

TABLE II Normalized Admittance Y/ $\mathrm{Y}$ of Loop Antennas in Dissipative Media . . . . . 20-21

TABLE III Normalized Admittance $Y / \Delta$ of Loop Antennas in Dissipative Media . . . . . 22-23

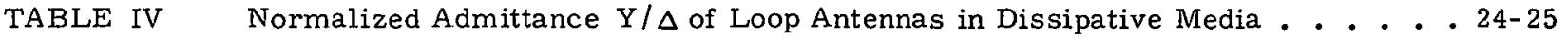

TABLE V Normalized Admittance $Y / \Delta$ of Loop Antennas in Dissipative Media . . . . . 26-27

TABLE VI Normalized Admittance Y/ $\Delta$ of Loop Antennas in Dissipative Media . . . . . 28-29 


\section{THE ADMITTANCE OF BARE CIRCULAR LOOP ANTENNAS IN A DISSIPATIVE IMEDIUM}

\section{Introduction}

The first general analysis of the circular loop as a transmitting antenna appears to be that of Hallen $^{3}$; he used the method of expansion in Fourier series. However, owing to the occurrence of a singularity or a very large value when the number of terms in the summation is sufficiently great, Hallen concluded that the series was divergent. Storer ${ }^{2}$ avoided the contribution from the large term by replacing the Fourier series by the corresponding integral and evaluating this in the sense of the Cauchy principal value. He provided extensive tables and graphs of the impedance, admittance, and diatribution of current for loups up to a wavelength in circumference with a number of different wire sizes. Recently, $\mathrm{Wu}^{3}$ re-examined the problem of evaluating the Fourier series. He considered Storer's expedient to be of doubtful validity and devised an alternative and improved method with approximations that are valid over larger ranges of the parameters.

Although formulated specifically for loops in air, the solutions of both Wu and Storer are readily applied to loops in an infinite homogeneous and isotropic medium by the introduction of the constitutive parameters of the medium at the appropriate points. It is the purpose of this paper to discuss the evaluation of the admittance of a loop antenna in an arbitrary dissipative medium from Wu's formula. A comparison with Storer's results is also provided.

Since loops up to a wave length in circumference are considered, the present work is a significant extension of the earlier studies by Kraichman ${ }^{4}$ and by Chen and King. ${ }^{5}$ Kraichman's analysis is based on a postulated uniform distribution of current around the loop which is valid only for electrically extremely small loops if these are bare or covered with a very thin layer of dielectric. The work of Chen and King makes use of Storer's analysis but retains only the first two terms in the Fourier Series. Although this is a considerably better approximation than that of Krajchman, it is also limited to electrically rather small loops. Indeed, even for loops with circumferences as small as $0.1 \lambda$ to $0.3 \lambda$, a surprisingly large error in the normalized conductance is made when only the first two terms in the Fourier series are retained.

\section{Analytical Formulation}

The admittance of a circular loop of radius $b$ when made of wire of radius a has been derived by Store ${ }^{2}$ and, in a somewhat more general form, by $\mathrm{Wu}^{3}$ specifically for antennas in air. The generalized formula for the normalized admittance $Y / \Delta=G / \Delta+j B / \Delta$ of a loop in an infinite homogenous and isotropic dissipative medium when driven by a delta-function generator is

$$
\frac{Y}{\Delta}=\frac{-j(1-j \alpha / \beta)}{\pi \zeta_{0}}\left[\frac{1}{a_{0}}+2 \sum_{1}^{\infty} \frac{1}{a_{n}}\right]
$$


where

$$
a_{n}=\frac{k b}{2}\left(K_{n+1}+K_{n-1}\right)-\frac{n^{2}}{k b} K_{n} .
$$

In this formula

$$
\begin{aligned}
& \mathrm{K}_{0}=\frac{1}{\pi} \ln \frac{8 \mathrm{~b}}{\mathrm{a}}-\frac{1}{2}\left[\int_{0}^{2 \mathrm{~kb}} \Omega_{0}(\mathrm{x}) \mathrm{dx}+\mathrm{j} \int_{0}^{2 \mathrm{~kb}} \mathrm{~J}_{0}(\mathrm{x}) \mathrm{dx}\right] \\
& \left.\mathrm{K}_{\mathrm{n}}=\mathrm{K}_{-\mathrm{n}}=\frac{1}{\pi}\left[\mathscr{H}\left(\frac{\mathrm{na}}{\mathrm{b}}\right) \mathscr{J}_{0}\left(\frac{\mathrm{na}}{\mathrm{b}}\right)+\mathrm{C}_{\mathrm{n}}\right]-\frac{1}{2} \int_{0}^{2 \mathrm{~kb}} s_{2 \mathrm{n}}(\mathrm{x}) \mathrm{dx}+\mathrm{j} \int_{0}^{2 \mathrm{~kb}} \mathrm{~J}_{2 \mathrm{n}}(\mathrm{x}) \mathrm{dx}\right] \\
& \mathrm{C}_{\mathrm{n}}=\ln (4 \mathrm{n})+0.5772 \ldots-2 \sum_{\mathrm{m}=0}^{\mathrm{n}-1}(2 \mathrm{~m}+1)^{-1}
\end{aligned}
$$

where $\mathscr{\Psi}_{0}(z)$ and $K_{0}(z)$ are the modified Bessel functions of the first and second kinds and $\Omega(x)$ is the Lommel-Weber function defined by

$$
\Omega_{m}(x)=\frac{1}{\pi} \int_{0}^{\pi} \sin (x \sin \theta-m \theta) d \theta
$$

In (2)

$$
\mathrm{k}=\beta-\mathrm{j} \alpha=\omega \sqrt{\mu(\epsilon-\mathrm{j} \sigma / \omega})=\omega \sqrt{\mu \epsilon_{\mathrm{o}}}[\mathrm{f}(\mathrm{p})-\mathrm{j} g(\mathrm{p})]
$$

is the complex propagation constant. In (7)

$$
p=\sigma / \omega \epsilon
$$

is the loss tangent of the medium and the $f(p)$ and $g(p)$ functions are defined as follow.s:

$$
f(p) \pm j g(p)=\sqrt{1 \pm j p}
$$

This is equivalent to

$$
f(p)=\cosh \left(\frac{1}{2} \sinh ^{-1} p\right), \quad g(p)=\sinh \left(\frac{1}{2} \sinh ^{-1} p\right) .
$$

The functions $f(p)$ and $g(p)$ are extensively tabulated in the literature. ${ }^{6}, ?$

In order to provide generally useful graphs and tables of the admittance of a loop anterua when immersed in a medium characterized by arbitrary values of $\sigma$ and $\epsilon$, it is convenient to introduce the normalizing factor

$$
\Delta=\sqrt{\frac{\epsilon_{r}}{\mu_{r}}} f(p)
$$

where $\epsilon_{\mathrm{r}}$ and $\mu_{\mathrm{r}}$ are the relative dielectric constant and permeability. This factor appears in (1); for antennas in air, it is equal to unity. 
As with a dipole antenna when center driven by a delta-function generator, the admittance $Y$ strictly does not exist, since its susceptance must become infinite owing to the knife-edge terminals with zero separation that characterize the delta-function generator. However, as shown for the dipole, ${ }^{8} \theta$ the representation of the current by continuous functions combined with the extreme localization of that part of the current that is associated with the knife edges of the generator, effectively omits the latter for thin wires unless a very large number of terms in the Fourier series is taken. If the infinite sum in (1) is replaced by a sum over a finite number of terms, an approximate formula is obtained that is a good measure of the admittance of the antenna for use with a practical method of driving when combined with a suitable terminal-zone network.

\section{Evaluation of the Tables}

The formula (1) was evaluated on a high-speed computer using successively $8,9,10,18,19$, and 20 terms in the series. The normalized conductance $G / \Delta$ and normalized susceptance $B / \Delta$ for $\alpha / \beta=0$ are shown in Fig. 1 as a function of the number of terms in the series. Curves are shown for $\beta \mathrm{b}=0,5$ and 2.0 and for $\Omega=2 \ln (2 \pi \mathrm{b} / \mathrm{a})=8,9,10,11$, and 12 . It is seen that the convergence is such that $\mathrm{G} / \Delta$ does not change noticeably for both values of $\beta \mathrm{b}$ and for all values of $\Omega$. On the other hand, $B / \Delta$ continues to increase with the number of terms. The rate of this increase is great when $\Omega<10$ and when $\beta \mathrm{b}$ is large; it is quite small for $\Omega \geq 10$, especially when $\beta \mathrm{b}$ is small. In general, it may be concluded that when $\Omega \geq 10,20$ terms in the Fourier series yield highly accurate values of $\mathrm{G} / \Delta$ for $\beta \mathrm{b} \leq 2.5$, quite good values of $B / \Delta$ for $\beta b<1$, and fair values when $1 \leq \beta b \leq 2.5$.

The values of $G / \Delta$ and $B / \Delta$ evaluated from Storer's theory ${ }^{2}$ are indicated on the right in Fig. 1. It is secn that they are in excellent agreement with Wu's results using 20 terms insofar as $G / \Delta$ is concerned, but that significant differences occur in $\mathrm{B} / \Delta$.

In Fig. 2 values of $B / \Delta$ are shown as functions of the number of terms in the Fourier series for $\beta b=2.0$ with $\Omega=8,12$, and 20 , when $\alpha / \beta$ is increased from zero to one. It is seen that except for $\alpha / \beta=1$, the curves for the relatively thick-wire loop with $\Omega=8$ or $2 \pi \mathrm{b} / \mathrm{a}=27: 3$ increase significantly with the number of terms. On the other hand, the curves for the thinner loops with $\Omega=12$ $(2 \pi \mathrm{b} / \mathrm{a}=259)$ and $\Omega=20(2 \pi \mathrm{b} / \mathrm{a}=11,013)$ are practically independent of the number of terms for all values of $\alpha / \beta$.

These results indicate that a Fourier series solution in which 20 terms are retained is satisfactory for determining the admittance of thin-wire loops $(\Omega \geq 10)$ that are not too large $(\beta \mathrm{b} \leq 2.5)$ when in air or an arbitrary dissipative medium. The approximation is excellent for the conductance, somewhat less accurate for the susceptance. Numerical values of the normalized admittance $Y / \Delta=$ $\mathrm{G} / \Delta+\mathrm{jB} / \Delta$ for loops with $\Omega=10,11,12,15,17.5$, and 20 are in the tables for $0 \leq \beta \mathrm{b} \leq 2.5$ and for $0 \leq \frac{\alpha}{\beta} \leq 1$ 


\section{Graphical Representation}

Graphs of the normalized conductance and susceptance of thin-wire loops as a function of the variable $\beta \mathrm{b}=2 \pi \mathrm{b} / \lambda$ are shown in Fig. 3-8. The parameter $\alpha / \beta$ of the surrounding medium ranges from zero to one. Note how insensitive to the size of the loop the admittance becomes as $\alpha / \beta$ approaches unity. This is true particularly of loops near antiresonance that have a high driving point impedance. Such an insensitivity merely means that most of the current has left the loop and has entered the surrounding dissipative medium.

\section{Conclusion}

The driving-point admittance of bare thin-wire loops up to a wavelength in circumference when immersed in an arbitrary dissipative medium has been determined from Wu's theory using 20 terms in the Fourier series. 


\section{LIST OF REFERENCES}

1. Hallen, E., "Theoretical Investigations Into Transmitting and Receiving Qualities of Antennae," Nova Acta Regiae Soc. Sci. Upsaliensis [4] 11, 1-44 (1938).

2. Storer, J. E., "Impedance of Thin-Wire Loop Antennas," Trans. Am. Inst. Elec. Engrs. 75, Part I, p. 606 (1956); also Cruft Laboratory Technical Report No. 212, $\overline{\text { May } 1,1955 .}$

3. Wu, T. T., "Theory of Thin Circular Loop Antenna," J. Math. Phys. 3, 1301-1304 (1962).

1. Kraishman, M. $R_{i}$, "Impedance of a Circular Loop in an Infinite Conducting Medium," J. Research Natl. Bur. Standards 660, pp. 499-503, July-August 1962.

5. Chen, C. L. and King, R. W. P., "The Small Bare Loop Antenna-Immersed in a Dissipative Medium," IEEE Trans. AP 11 (to be published).

6. King, R. W. P., "Fundamental Electromagnetic Theory, "Appendix II, Dover Publications (1963).

7. Gooch, D. W., Harrison, C. W. , Jr., King, R. W. P., and Wu, T. T., "Impedances and Admittances of the Long Antenna in Air and in Dissipative Media With Tables of the Functions $f(p) \pm i g(p)=\sqrt{1 \pm i p}$, "Cruft Laboratory Technical Report No. 352, January 15,1962 .

8. Wu, T. T. and King, R. W. P., "Driving Point and Input Admittance of Linear Antennas," J. Appl. Phys. 30, p. 76 (1959).

9. Duncan, R. H. and Hinchey, F. A., "Cylindrical Antenna Theory, " J. Research Natl. Bur. Standards 64D, pp. 569-584, September-October 1960. 


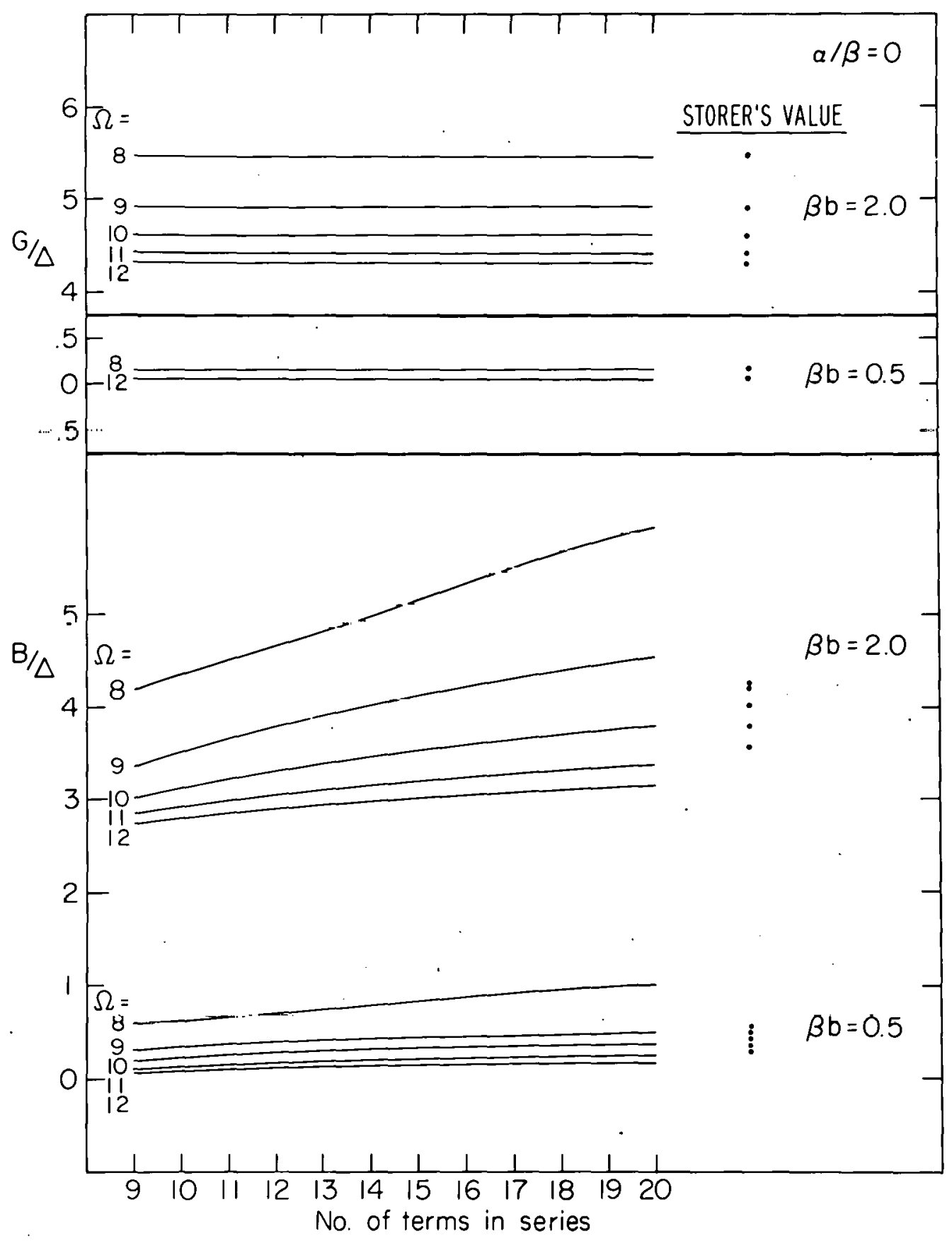

Fig. 1 Normalized conductance and susceptance of circular loop in air.as a function of the number of terms - Wu's theory for $\beta b=0.5$ and 2.0 , $\Omega=2 \ln \left(\frac{2 \pi b}{a}\right)=8-12$ 


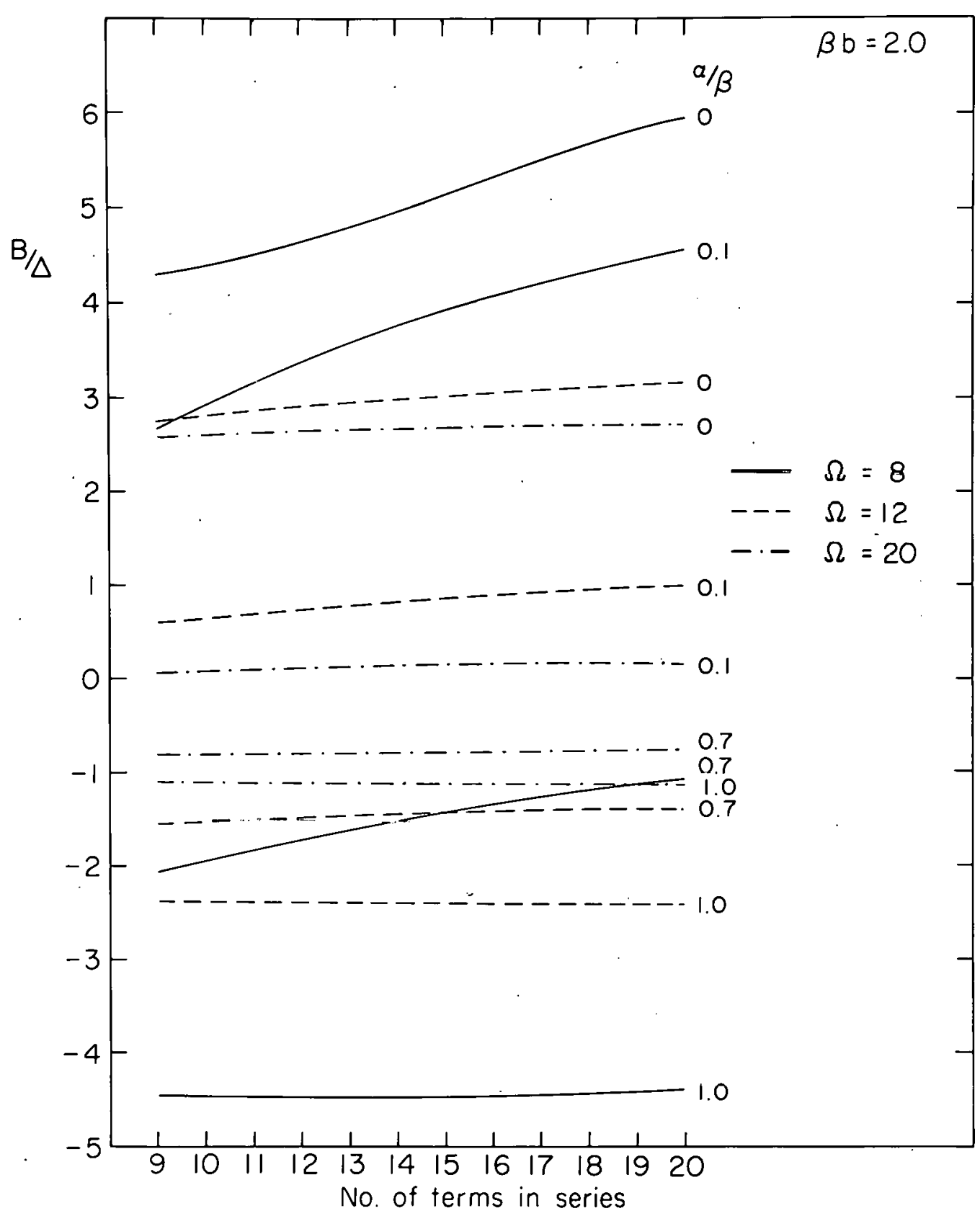

Fig. 2 Normalized susceptance of circular loop in a dissipative medium with propagation constant $\mathrm{k}=\beta-\mathrm{j} \alpha$ as a function of the number of terms $\mathrm{Wu}$ 's theory for $\beta \mathrm{b}=2.0, \Omega=8,12,20, \alpha / \beta=0-1$. 


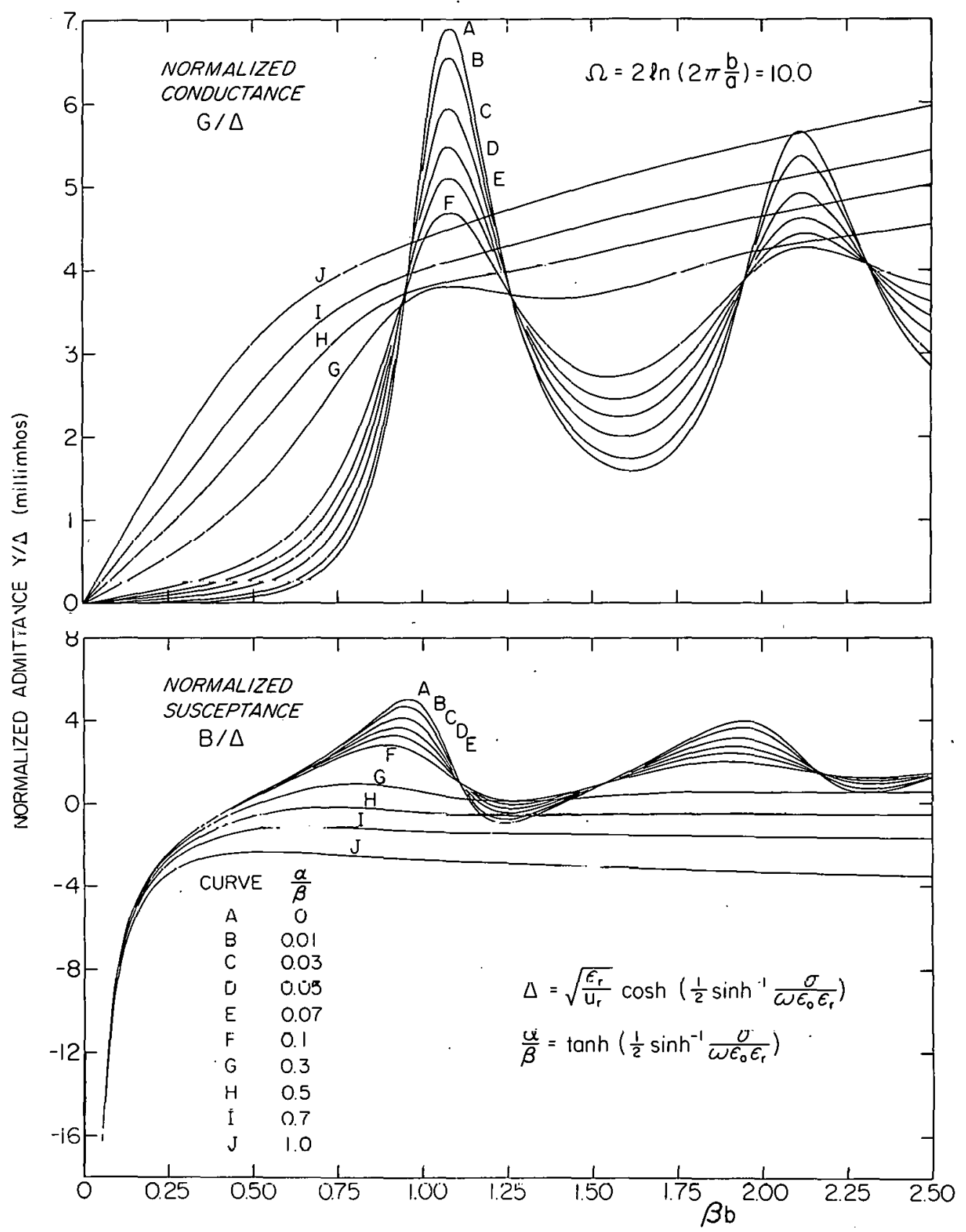

Fig. 3 Normalized admittance of circular loop antenna in a dissipative medium - Wu's theory, $\Omega=10$ 


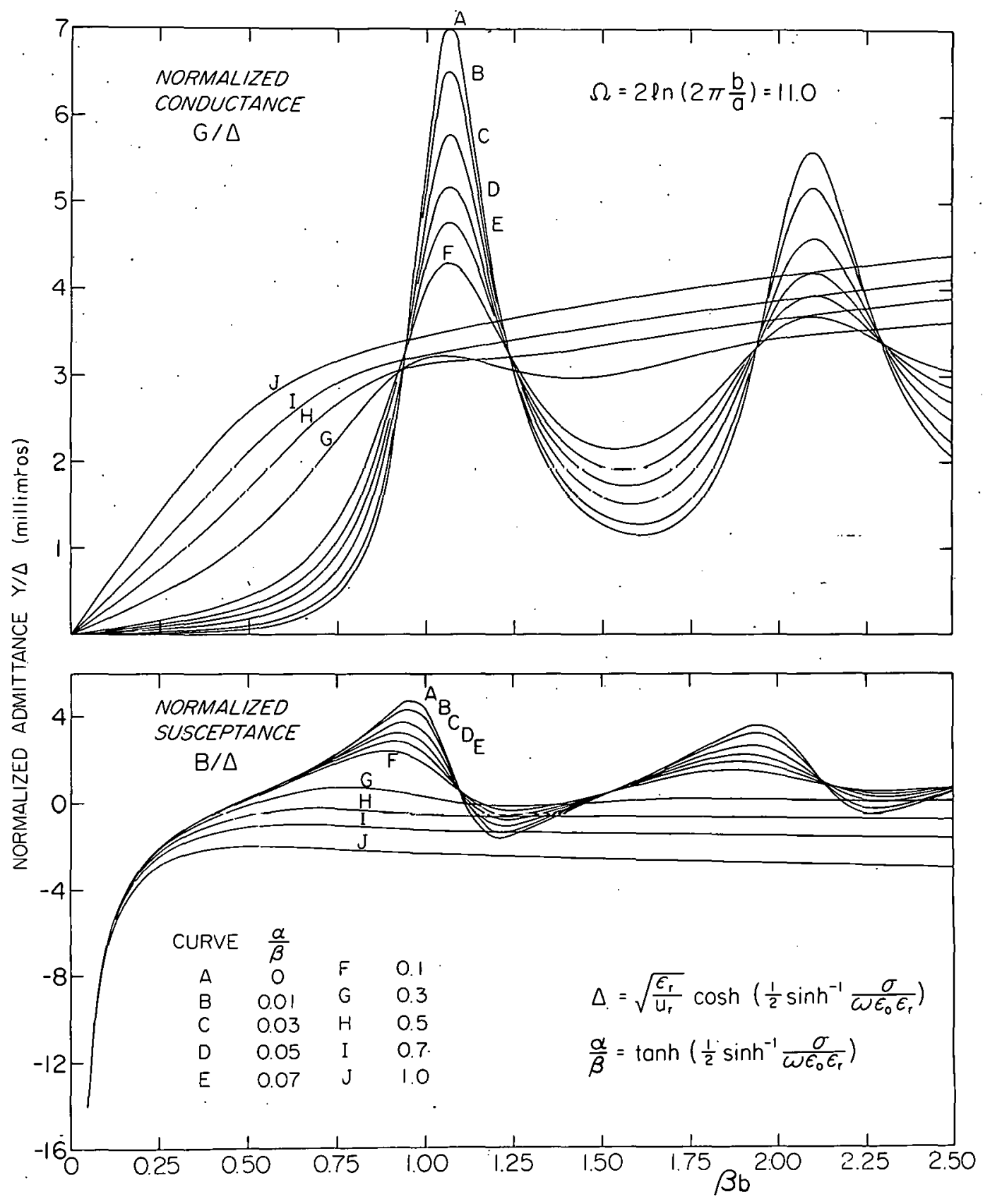

Fig. 4 Normalized admittance of circular loop antenna in a dissipative medium - Wu's theory, $\Omega=11$ 


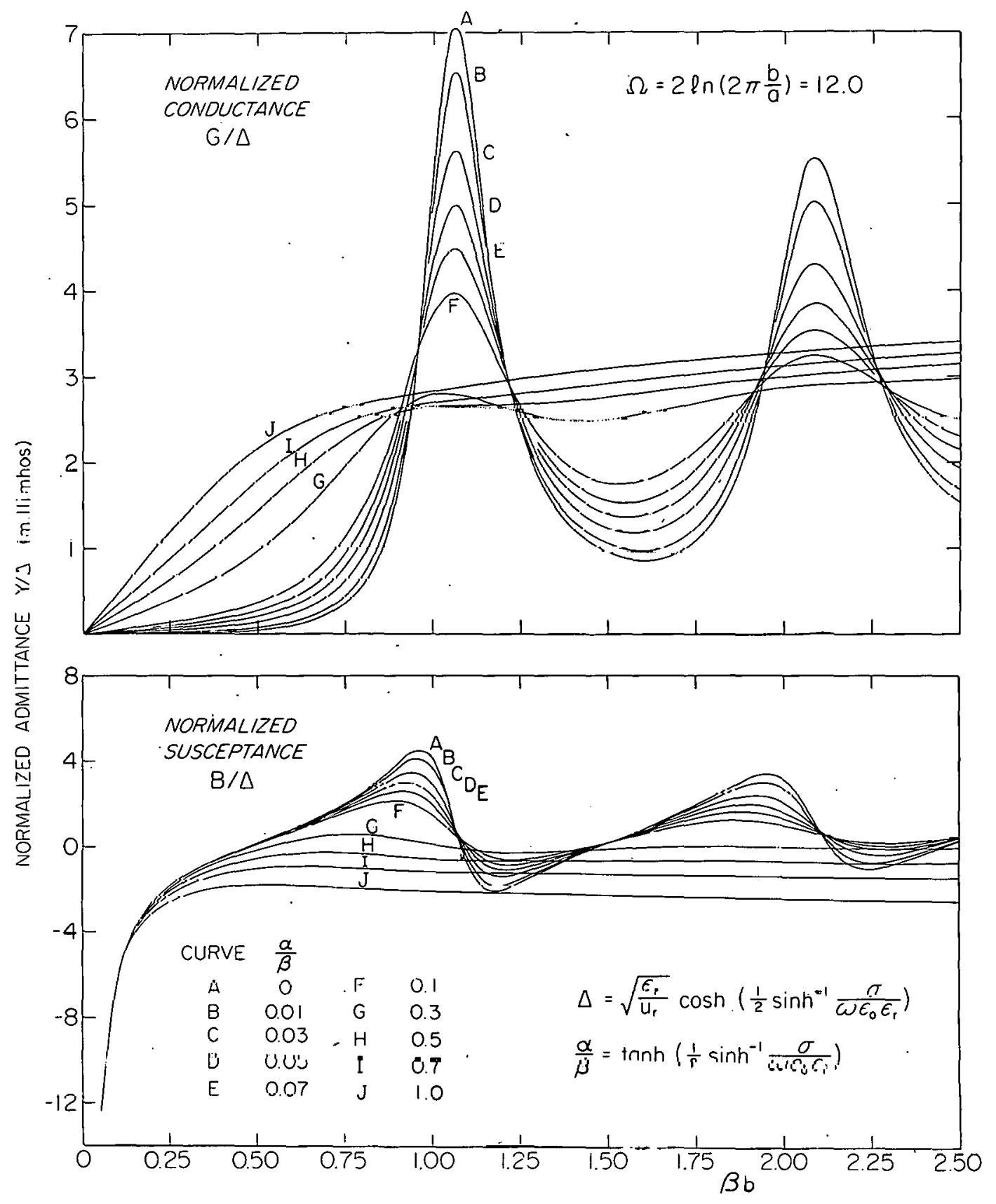

Fig. 5 Normalized admittance of circular loop antenna in a dissipative medium - Wu's theory, $\Omega=12$. 


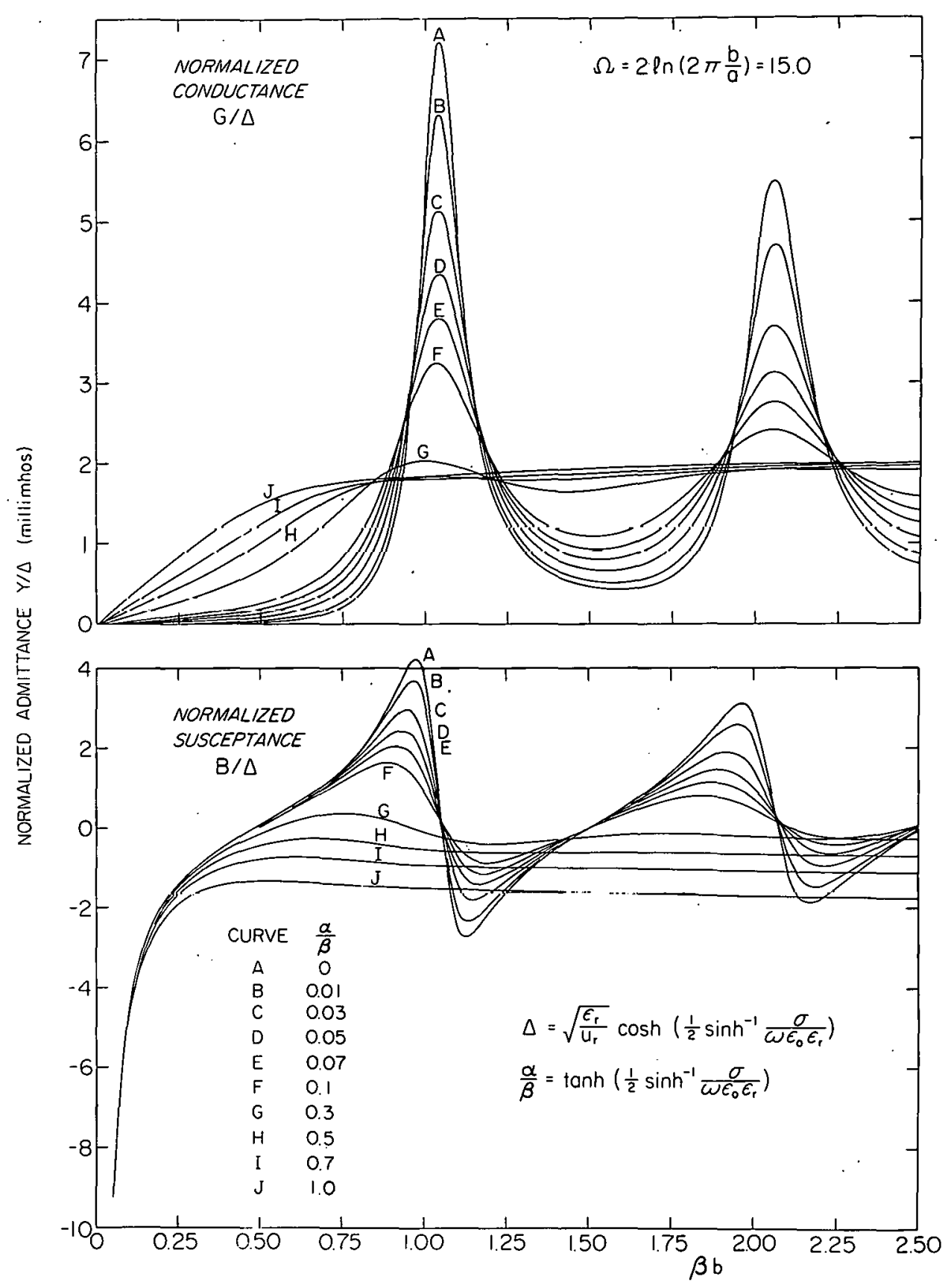

Fig. 6 Normalized admittance of circular loop antenna in a dissipative medium - Wu's theory, $\Omega=15$ 


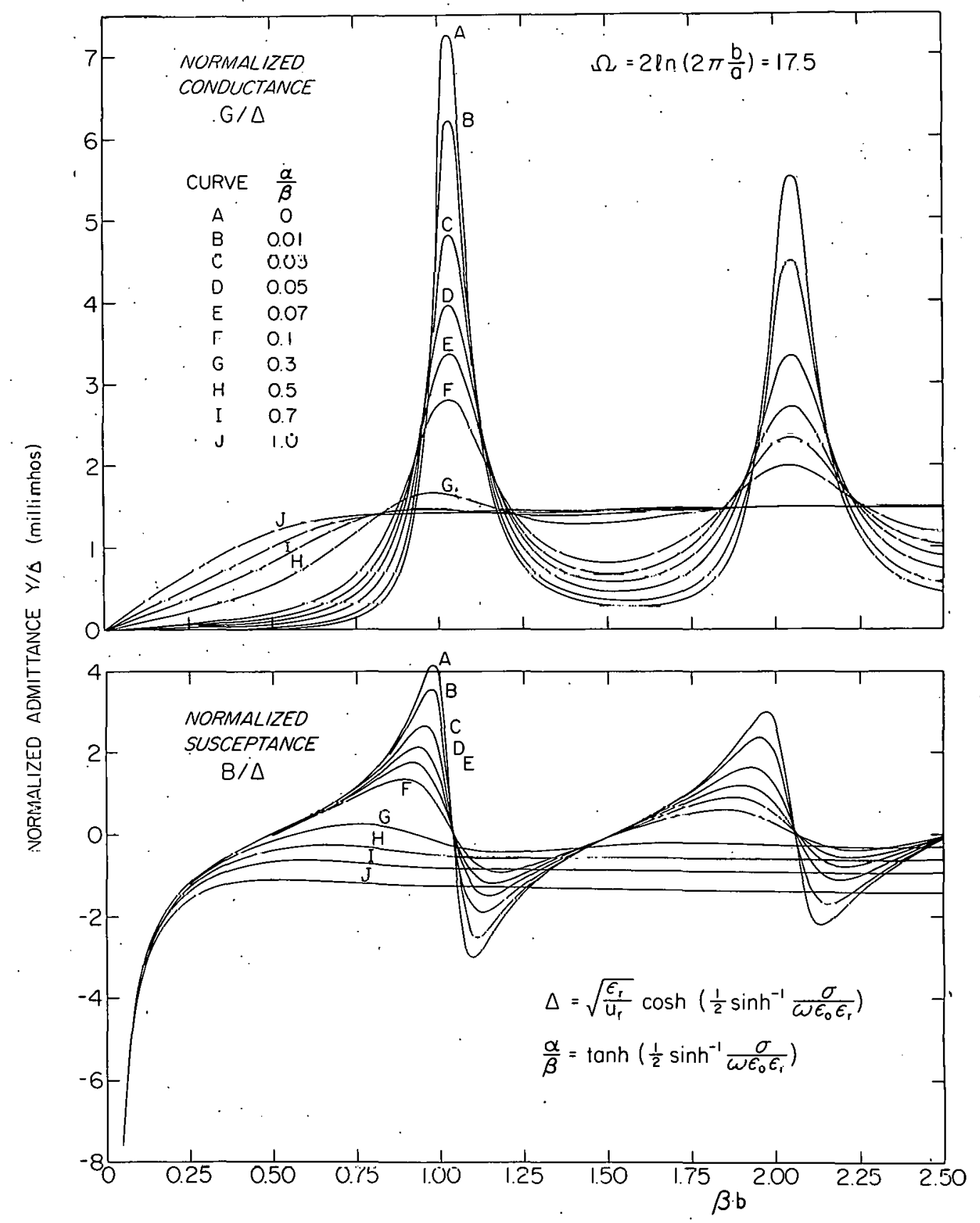

Fig. 7 Normalized admittance of circular loop antenna in a dissipative medium - Wu's theory, $\Omega=17.5$ 


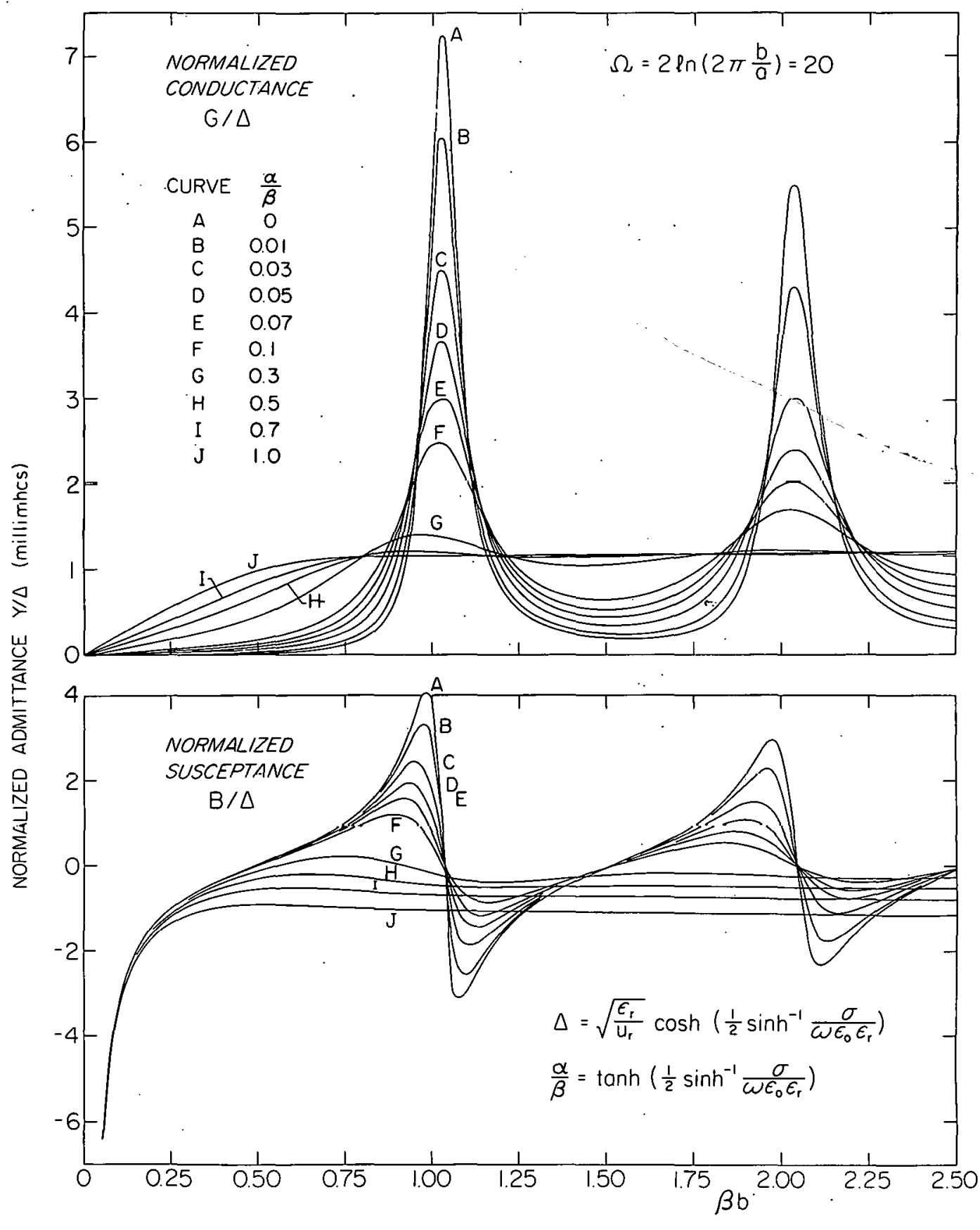

Fig. 8 Normalized admittance of circular loop antenna in a dissipative medium - Wu's theory, $\Omega=20$ 
TABLE I

Normalizec Admittance $Y / \Delta$ of Loop Antennas in Diss :pative Med:a

$\beta b$

$$
\begin{gathered}
\frac{\alpha}{\beta}=0.00 \\
Y / \Delta
\end{gathered}
$$

0.05

0.10

0.15

0.20

0.25

0.35

0.40

0.45

0.50

0.55

0.60

0.65

0.70
0.75

0.80

0.85

0.90
0.95

1.00

1.05

1.10
1.15

1.15
1.20

1.25

1.30

1.35

1.40

1.45
1.50

1.55
1.60

1.55
1.65

1.65

1.70

1.75
1.80

1.85

1.90

1.95

2.00

2.05

2.10

2. 15

2.20

2.25

2.30

2. 35

2.40

2.45

2.50
$0.0003-16.1742$

$0.0014-7.8236$

$0.0032-4.9201$

$0.0061-3.3750$

$0.0102-2.3691$

$0.0162-1.6283$

$0.0248-1.0333$

$0.0370-0.5233$

$0.0546-0.0624$

0.08010 .3730

$\begin{array}{ll}0.1174 & 0.8006 \\ 0.1728 & 1.2351\end{array}$

$0.2561 \quad 1.0908$

0.38372 .1816

$0.5830 \quad 2.7214$

$0.9009 \quad 3.3195$

1.41613 .9671

$2.2513 \quad 4.5965$

$\begin{array}{ll}3.5409 & 4.9903\end{array}$

$6.6359 \quad 3.2896$

$6.8730 \quad 1.3221$

$0.0398-0.1400$

$4.9127-0.7848$

$3.9355-0.8825$

$3.1945-0.7081$

$2.6537-0.4132$

$2.2627-0.0671$

$\begin{array}{ll}1.9826 & 0.3014\end{array}$

1.7878

0.681 .9

$1.6637 \quad 1.0722$

1.6039

1.6877

1.8540

1306

2. 5444

3.1165
3.8359

4.6171

5.2801

5.6233

5.5686
5.2098

4.7102

4.1983

3.7412

3.3624

3.0648

2.8436

1.4734

2.3137

2.7491

3. 1797

3.5744
3.8733

3.9815

$3.790 t$

3. $253 \mathrm{C}$

2.4710

$1.671 E$
$1.056 \mathrm{C}$

0.6964

0.5656

0.6027

0.7520

0.9739
1.2429 $\frac{\alpha}{\beta}=0.01$

$Y / \Delta$

$\Omega=10$

$\frac{\alpha}{\beta}=0.03$

$\mathrm{Y} / \Delta$

$0.0038-16.1742$

$0.0084-7.8237$

$0.0139-4.9203$

$0.0206-3.3752$

$0.0288-2.3696$

$0.0392-1.6290$

$0.0526-1.0345$

$0.0702-0.5250$

$0.0939-0.0651$

$0.1266 \quad 0.3688$

$\begin{array}{ll}0.1724 & 0.7939 \\ 0.2380 & 1.2247\end{array}$

$0.2380 \quad 1.2247$

$0.3337 \quad 1.6740$

$\begin{array}{ll}0.4765 & 2.1545 \\ 0.6938 & 2.6768\end{array}$

$1.0313 \quad 3.2450$

$1.5620 \quad 3.8414$

2.39024 .3886

$3.6073 \quad 4.6755$

5.1043

4.3157

6.30113 .0461

$6.5054 \quad 1.3362$

$5.8108 \quad 0.0327$

$4.8271-0.5374$

$3.9399-0.7158$

$3.2478-0.5303$

$2.7340-0.3188$

2.35950 .0008

$2.0916 \quad 0.3478$

$1.9076 \quad 0.7098$

$1.7942 \quad 1.0822$

1.7457

1. 7628

1.4644

2.2545

$2.3038 \quad 3.0368$

$2.7011 \quad 3.3725$

$3.2271 \quad 3.6068$

$3.8587 \quad 3.6637$

$4.5146 \quad 3.4690$

$5.0531 \quad 3.0048$

$5.3316 \quad 2.3600$

$5.2987 \quad 1.7030$

$5.0200 \quad 1.1830$

$\begin{array}{ll}4.6146 & 0.8631 \\ 4.1841 & 0.7327\end{array}$

$3.7886 \quad 0.7500$

$3.4543 \quad 0.8714$

$3.1888 \quad 1.0632$

$\begin{array}{ll}3.1888 & 1.0632 \\ 2.9916 & 1.3016\end{array}$
$0.0108-16.1744$

$\begin{array}{lll}0.0225 & -7.8241\end{array}$

$0.0353-4.9209$

$0.0496-3.3763$

$0.0660-2.3711$

$0.0851-1.6313$

$0.1081-1.0378$

$0.1364-0.5300$

$0.1723-0.0723$

0.21910 .3580

0.28140 .7775

$0.3666 \quad 1.1997$

$0.4857 \quad 1.0354$

0.65632 .093

0.90512 .5795

$\begin{array}{ll}1.2738 & 3.0877 \\ 1.8222 & 3.5873\end{array}$

$2.6194 \quad 3.9903$

$3.6909 \quad 4.11131$

$4.8814 \quad 3.6941$

$5.7564 \quad 2.6416$

$5.9058 \quad 1.3233$

$\begin{array}{rr}5.4080 & 0.2831 \\ 4.0528 & -0.2693\end{array}$

$3.9256-0.4304$

$3.3294-0.3531$

$2.8724-0.1475$

2.53430 .1243

2.29290 .4306

2.13110 .7558

$2.0383 \quad 1.0923$

$2.0098 \quad 1.4359$

$2.0457 \quad 1.7829$

$2.1504 \quad 2.1276$

$2.3315 \quad 2.459 .4$

$2.5975 \quad 2.7605$

2.95313 .0020

3.39023 .143 .5

3.8765 .3 .1400

4.3470

4.7145

4.9046

4.8948

4.4510

4.1477

3.8563

3.6021

3.3970
3.2453

2.6125

2. 1627

1. 7088

1.3374

0.9786

0.0755

1.0578

1. 2017

1. 3880 $\frac{\alpha}{\beta}=0.05$

$Y / \Delta$

$$
\frac{\alpha}{\beta}=0.07
$$

$0.0178-16.1747$

$0.0366-7.8247$

$0.0567-4.9220$

$0.0787-3.3779$

$0.1031-2.3735$

$0.1309-1.6346$

$0.1634-1.0426$

$0.2024-0.5367$

$0.2502 \quad-0.0819$

$0.3107 \quad 0.3442$

$0.3889 \quad 0.7574$

$\begin{array}{ll}0.4926 & 1.1698 \\ 0.6333 & 1.5903\end{array}$

0.63332 .5903

$\begin{array}{ll}0.8282 & 2.0249 \\ 1.1027 & 2.4733\end{array}$

1.49292 .9229

2.04413 .3345

$2.7957 \quad 3.6205$

$\begin{array}{ll}3.7295 & 3.6295 \\ 4.6828 & 3.1964\end{array}$

$5.3366 \quad 2.3176$

$5.4445 \quad 1.2783$

$5.0746 \quad 0.4422$

$4.4874-0.0365$

$3.8932-0.2040$

$3.3857-0.1640$

$2.9860-0.0016$

2.68650 .2295

2.47310 .4985

$2.3338 \quad 0.7885$

$2.2603 \quad 1.0898$

$2.2484 \quad 1.3956$

$2.2976 \quad 1.6998$

$2.4098 \quad 1.9945$

$2.8340 \quad 2.5028$

$3.1440 \quad 2.6759$

$3.5030 \quad 2.7596$

$3.8793 \quad 2.7291$

$4.2248 \quad 2.5745$

$4.4854 \quad 2.3133$

$4.6209 \quad 1.9919$

$4.6219 \quad 1.6713$

$4.5110,1.4048$

$\begin{array}{ll}4.3285 & 1.2227\end{array}$

$3.9044 \quad 1.1231$

$3.7163 \quad 1.1816$

$3.5633 \quad 1.2908$

$\begin{array}{ll}3.4515 & 1.4360\end{array}$

$0.0248-16.1751$

$0.0507-7.8257$

$0.0781-4.9236$

$0.1076-3.3802$

$0.1401-2.3767$

$0.1766-1.6391$

$0.2186-1.0487$

$0.2680-0.5452$

$0.3276-0.0938$

$0.4013 \quad 0.3274$

$0.4947 \quad 0.7335$

$0.6158 \quad 1.1350$

$0.7761 \quad 1.5393$

$0.9921 \quad 1.9491$

$1.2868 \quad 2.3599$ 
TABLE I

Normalized Admittance $\mathrm{Y} / \Delta$ of Loop Antennas in Dissipative Media

Eb $\frac{\alpha}{\beta}=0.10$

$\mathrm{Y} / \Delta$

0.05

0.10
0.15

0.15

0.25

0.30

0.35

0.40

0.45
0.50

0.55

0.60

0.65

0.70

0.75

0.80

0.85

0.90

1.00

1.05

1.05
1.10

1.15
1.20

1.25

1.30

1.35

1.40

1.45
1.50

1.55

1.55

1.60

1.70

1.75

1.80

1.85

1.90

1.95

2.05

2.10

2.15

2.20

2.25

2.30

2.35

2.40

2.45

2.50 $\frac{\alpha}{\beta}=0.30$

$Y / \Delta$

$0.1052-16.1903$

$0.2123-7.8572$

$0.3228-4.9733$

$0.4382-3.4511$

$0.5604-2.4731$

$0.6914-1.7670$

$0.8334-1.2165$

$0.9892-0.7645$

$1.1614-0.3806$

$1.3530-0.0485$

$\begin{array}{ll}1.5664 & 0.2395\end{array}$

1.80330 .4853

$2.0634 \quad 0.6864$

$2.3432 \quad 0.8372$

$2.6350 \quad 0.9311$

$\begin{array}{ll}2.9256 & 0.9628 \\ 3.1976 & 0.9319\end{array}$

$3.4323 \quad 0.8458$

$3.6142 \quad 0.7204$

$3.7357 \quad 0.5770$

$3.7992 \quad 0.4377$

$3.8155 \quad 0.3199$

3.7994

3.7664

$3.7293 \quad 0.1644$

$3.6760 \quad 0.1731$

$\begin{array}{ll}3.6769 & 0.2024 \\ 3.6706 & 0.2454\end{array}$

$3.6795 \quad 0.2962$

$3.7030 \quad 0.3496$

$\begin{array}{ll}3.7396 & 0.4014\end{array}$

$3.7869 \quad 0.4484$

$3.8422 \quad 0.4885$

$3.9028 \quad 0.5203$

$3.9657 \quad 0.5434$

$4.0284 \quad 0.5582$

$4.0887 \quad 0.5656$

$4.1451 \quad 0.5671$

$\begin{array}{ll}4.1964 & 0.5645 \\ 4.2423 & 0.5594\end{array}$

$4.2829 \quad 0.5537$

$4.3189 \quad 0.5486$

$4.3510 \quad 0.5451$

$4.3804 \quad 0.5440$

$4.4080 \quad 0.5453$

4.43490 .5491

$\begin{array}{ll}4.4617 & 0.5551 \\ 4.4891 & 0.5628\end{array}$

$4.5175 \quad 0.5717$

$\begin{array}{ll}4.5175 & 0.5717 \\ 4.5471 & 0.5811\end{array}$ $\Omega=10$

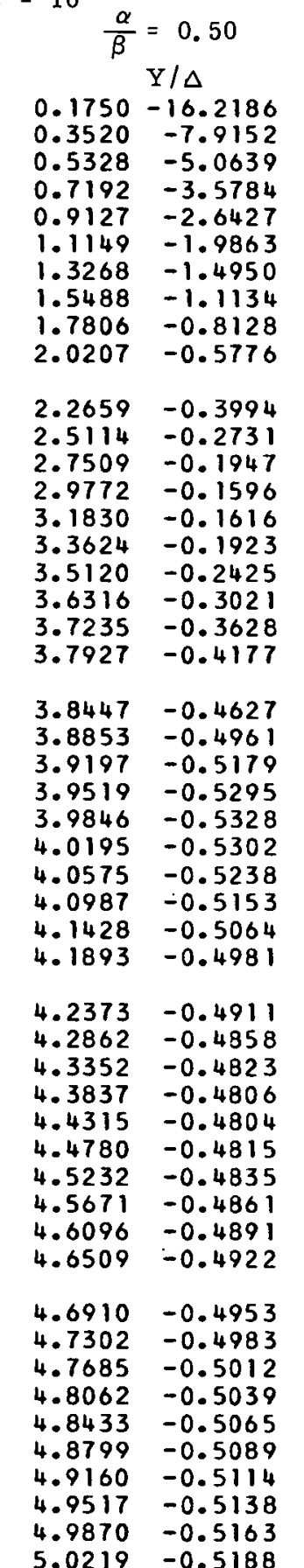

$\frac{\alpha}{\beta}=1.00$ $\mathrm{Y} / \Delta$

$0.3485-16.3505$

$0.6954-8.1837$

$1.0399-5.4773$

$1.3802-4.1482$

$1.7135-3.3821$

$2.0360-2.9087$

$2.3432-2.6117$

$2.6307-2.4314$

$2.8944-2.3322$

$3.1316-2.2904$

$3.3411-2.2880$

$3.5237-2.3117$

$3.6816-2.3511$

$3.8180-2.3985$

$3.9368 \quad-2.4487$

$4.0416 \quad-2.4985$

$4.1357-2.5462$

$4.2219-2.5909$

$4.3023-2.6327$

$4.3784-2.6718$

$\begin{array}{ll}4.4513 & -2.7087\end{array}$

$4.5218 \quad-2.7437$

$4.5902-2.7774$

$\begin{array}{ll}4.6568 & -2.8099\end{array}$

$4.7218 \quad-2.8417$

$4.7852-2.8728$

$4.8472-2.9034$

$4.9077-2.9336$

$\begin{array}{ll}4.9669 & -2.9633 \\ 5.0246 & -2.9927\end{array}$

$5.0811-3.0217$

$5.1363-3.0503$

$5.1903-3.0785$

$5.2431-3.1064$

$5.2949-3.1339$

$5.3455-3.1610$

$5.3952-3.1878$

$5.4438-3.2142$

$5.4916-3.2403$

$5.5384-3.2661$

$5.5844-3.2915$

$5.6295-3.3166$

$5.6739-3.3414$

$5.7174-3.3659$

$5.7602-3.3900$

$5.8023-3.4139$

$5.8437-3.4375$

$5.8844-3.4607$

$5.9245-3.4837$

$5.9639-3.5064$ 
TABLE II

Normalizec Admitiance $\mathrm{Y} / \Delta$ of Loop Antennas in Lissipative M

$\beta \mathrm{b}$

$$
\begin{gathered}
\frac{\alpha}{\beta}=0.00 \\
\mathrm{Y} / \Delta
\end{gathered}
$$

0.05

0.10

0.15

0.20

0.25

0.30

0.35

0.40

0.55

0.55

0.60

0.65

0.70

0.75
0.80

0.85

0.90

0.95
1.00

1.05

1.05
1.10

1.10
1.15

1.15
1.20

1.25

1.30

1.35

1.40

1.45

1.50

1.55

1.60

1.65

1.70

1.75

1.80
1.85

1.90

1.95

2.00

2.05

2.10

2. 15

2.20

2.25

2.30

2.35

2.40

2.45

2.50
$0.0002-14.0300$

$0.0010-6.7984$

$0.0024-4.2891$

$0.0046 \quad-2.9573$

$0.0077-2.0931$

$0.0122-1.4585$

$0.0187-0.9503$

$0.0279-0.5154$

$0.0413-0.1228$

$0.0606 \quad 0.2483$

$0.0890 \cdot 0.6135$

$0.1314 \quad 0.9867$

$0.1958 \quad 1.3813$

$0.2957 \quad 1.8124$

$0.4550 \quad 2.2968$

$0.7169 \quad 2.8520$

$\begin{array}{ll}1.1622 & 3.4865 \\ 1.9374 & 4.1612\end{array}$

3.26224 .6662

$5.1932 \quad 4.3834$

$6.8232 \cdot 2.5879$

$0.7120 \quad 0.1681$

$5.3779-1.2319$

$4.0411-1.5838$

$3.0663-1.4420$

$2.4031-1.1269$

$1.9519-0.7638$

$1.6408-0.3945$

$1.4256-0.0298$

1.28040 .3306

$\begin{array}{ll}1.1914 & 0.6913 \\ 1.1531 & 1.0581\end{array}$

$\begin{array}{ll}1.1531 & 1.0581 \\ 1.1069 & 1.4370\end{array}$

$1.1609 \quad 1.4370$

$1.2412 \quad 1.8330$

$1.6496 \quad 2.6778$

$2.0527 \quad 3.0985$

$2.6507 \quad 3.4504$

$3.4702 \quad 3.6083$

$4.4330 \quad 3.3816$

$5.2599 \quad 2.6373$

$5.5806 \quad 1.5398$

$5.2983 \quad 0.5189$

$4.6658-0.1268$

$3.9731-0.3895$

$3.3679-0.3895$

$2.8872-0.237$

$2.5221-0.0053$

$\begin{array}{ll}2.2533 & 0.2702 \\ 2.0636 & 0.5696\end{array}$
$\Omega=11$

$$
\frac{\alpha}{\beta}=0.01 \quad \Omega=11 \frac{\alpha}{\beta}=0.03 \quad \frac{\alpha}{\beta}=0.05
$$$$
\mathrm{Yi \Delta}
$$

$0.0031-14.0300$

$0.0068-6.7985$

$0.0112-4.2892$

$0.0165-2.9576$

$0.0230-2.0935$

$0.0312-1.4590$

$0.0417-0.9511$

$0.0555-0.5168$

$0.0741-0.1249$

$0.0996 \quad 0.2450$

$0.1354 \quad 0.6084$

0.18690 .9785

$0.2625 \quad 1.3682$

$0.3767 \quad 1.7908$

0.55412 .2605

$0.8379 \quad 2.7892$

1.30523 .3748

$2.0856 \quad 3.9612$

$\begin{array}{ll}3.3433 & 4.3304 \\ 5.0403 & 3.9540\end{array}$

$6.3748 \quad 2.3528$

6.29250 .2928

$5.1883-0.9740$

$4.0110-1.3585$

$3.1097-1.2781$

$2.4775-1.0124$

$2.0401-0.6842$

$\begin{array}{ll}1.7368 & -0.3397\end{array}$

$1.5278,0.0063$

$1.3894 \quad 0.3513$

$1.3085 \quad 0.6976$

$1.2801 \quad 1.0490$

$1.3055 \quad 1.4093$

$1.3927 \quad 1.7811$

$\begin{array}{ll}1.5567 & 2.1629\end{array}$

$1.8213 \quad 2.5447$

$2.2174 \quad 2.8991$

$2.7748 \quad 3.1669$

$3.4925 \quad 3.2432$

$4.2812 \quad 2.9952$

$4.9228 \quad 2.3608$

$5.1708 \quad 1.4821$

$4.9680 \quad 0.6572$

$4.4801 \quad 0.1015$

$3.9146-0.1562$

$2.9690-0.0792$

$2.6370-0.0792$

$2.0370-0.1138$

$\begin{array}{ll}2.3898 & 0.3544 \\ 2.2157 & 0.6228\end{array}$
$\mathrm{Y} / \Delta$

$0.0089-14.0332$

$0.0184-6.7938$

$0.0288-4.2877$

$0.0405-2.9584$

$0.0537-2.0947$

$0.0692-1.4609$

$0.0877-0.9538$

$0.1106-0.5207$

$0.1395-0.1306$

0.17710 .2365

$0.2274 \quad 0.5955$

$\begin{array}{ll}0.2963 & 0.9587 \\ 0.3934 & 1.3372\end{array}$

$\begin{array}{ll}0.3934 & 1.3372 \\ 0.5341 & 1.7413\end{array}$

0.74342 .1796

$1.0628 \quad 2.6537$

$1.5590 \quad 3.1444$

$2.3258 \quad 3.5737$

$\begin{array}{ll}3.4364 & 3.7337 \\ 4.7489 & 3.2605\end{array}$

$5.6666 \quad 1.9755$

$5.6112 \quad 0.4414$

$4.8322-0.5849$

$3.9190-0.9827$

$3.1599-0.9866$

$2.5967-0.8009$

$2.1941-0.5346$

$1.9114-0.2367$

1.71790 .0725

1.59410 .3860

$1.5289 \quad 0.7021$

$\begin{array}{ll}1.5178 & 1.0211\end{array}$

$1.5618 \quad 1.3432$

$1.6671 \quad 1.6063$

$1.8446 \quad 1.9837$

$\begin{array}{ll}2.1085 & 2.2804 \\ 2.4729 & 2.5267\end{array}$

$2.9409 \quad 2.6739$

$3.4849 \quad 2.6559$

$4.0239 \quad 2.4143$

$4.4283 \quad 1.9503$

$4.5834 \quad 1.3631$

$4.4695 \quad 0.8083$

$\begin{array}{ll}4.1672 & 0.4039 \\ 3.7873 & 0.1840\end{array}$

$3.4122 \quad 0.1236$

$3.0846 \quad 0.1794$

$2.8196 \quad 0.3125$

$2.6184 \quad 0.4740$

$2.4776 \quad 0.7372$
$\mathrm{Y} / \Delta$

$0.0146-14.0304$

$0.0300-6.7993$

$0.0465-4.2906$

$0.0644 \quad-2.9597$

$0.0843-2.0966$

$0.1071-1.4636$

$0.1336-0.9576$

$0.1654-0.5261$

$0.2045-0.1384$

$0.2540 \quad 0.2253$

$0.3181 \quad 0.5792$

0.4036 .0 .934

$0.5206 \quad 1.3002$

$0.6847 \quad 1.6840$

$0.9205 \quad 2.0890$

$\begin{array}{ll}1.2655 & 2.5085\end{array}$

$1.7735 \quad 2.9108$

$2.5042 \cdot 3.2098$

$3.4678 \quad 3.2271$

$5.1373 \quad 1.6845$

$5.0905 \quad 0.5083$

$4.5203 \quad-0.3207$

$3.8074 \quad-0.694$

$3.175 t-0.7445$

$2.6836-0.6166$

$2.321 E-0.4011$

1.88910 .1291

$1.7808 \quad 0.411$

$\begin{array}{lll}1.730 \hat{2} & 0.6964\end{array}$

$1.7335 \quad 0.9823$

$1.7904 \quad 1.2661$

$2.084 . \quad 1.8024$

$2.3332 \quad 2.0290$

$2.6540 \quad 2.1965$

$3.0377 \quad 2.2698$

$3.4490 \quad 2.2115$

3.8269

2.0010

$4.0950 \quad 1.6577$

$4.1968 \quad 1.2493$

$4.1275 \quad 0.8645$

$3.931 ? \quad 0.5719$

$3.6720 \quad 0.3983$

0.3368

0.3649

0.458

$\begin{array}{ll}2.7980 & 0.5957 \\ 2.6894 & 0.7619\end{array}$

$$
\frac{\alpha}{\beta}=0.07
$$

$\mathrm{Y} / \Delta$

$0.0204-14.0308$

$0.0416-6.8001$

$0.0641-4.2919$

$0.0883-2.9616$

$0.1149-2.0992$

$0.1449-1.4672$

$0.1793-0.9626$

$0.2200-0.5330$

$0.2691-0.1481$

$0.3300 \quad 0.2116$

$\begin{array}{ll}0.4075 & 0.5595\end{array}$

$0.5086 \quad 0.9057$

$0.6437 \quad 1.2577$

$0.8283 \quad 1.6198$

$.0853 \quad 1.9908$
.4469

1.44692 .3570

$\begin{array}{ll}1.9532 & 2.6796 \\ 2.6350 & 2.8737\end{array}$

$3.4625 \quad 2.7970$

$4.2623 \quad 2.3094$

$\begin{array}{ll}4.7312 & 1.4514\end{array}$

$4.6867 \quad 0.5287$

$4.2556-0.1428$

$3.6936-0.4765$

$3.1707-0.5487$

$2.7473-0.4611$

$2.4275-0.2863$

$2.0422-0.0667$

$\begin{array}{ll}2.0422 & 0.1749 \\ 1.9497 & 0.4266\end{array}$

$1.9125 \quad 0.6814$

$1.9270 \quad 0.9348$

$1.9924 \quad 1.1818$

$2.1102 \quad 1.4155$ 
TABLE II

Normalized Admittance $Y^{\prime} \Delta$ of Loop Antennas in Dișsipative Media

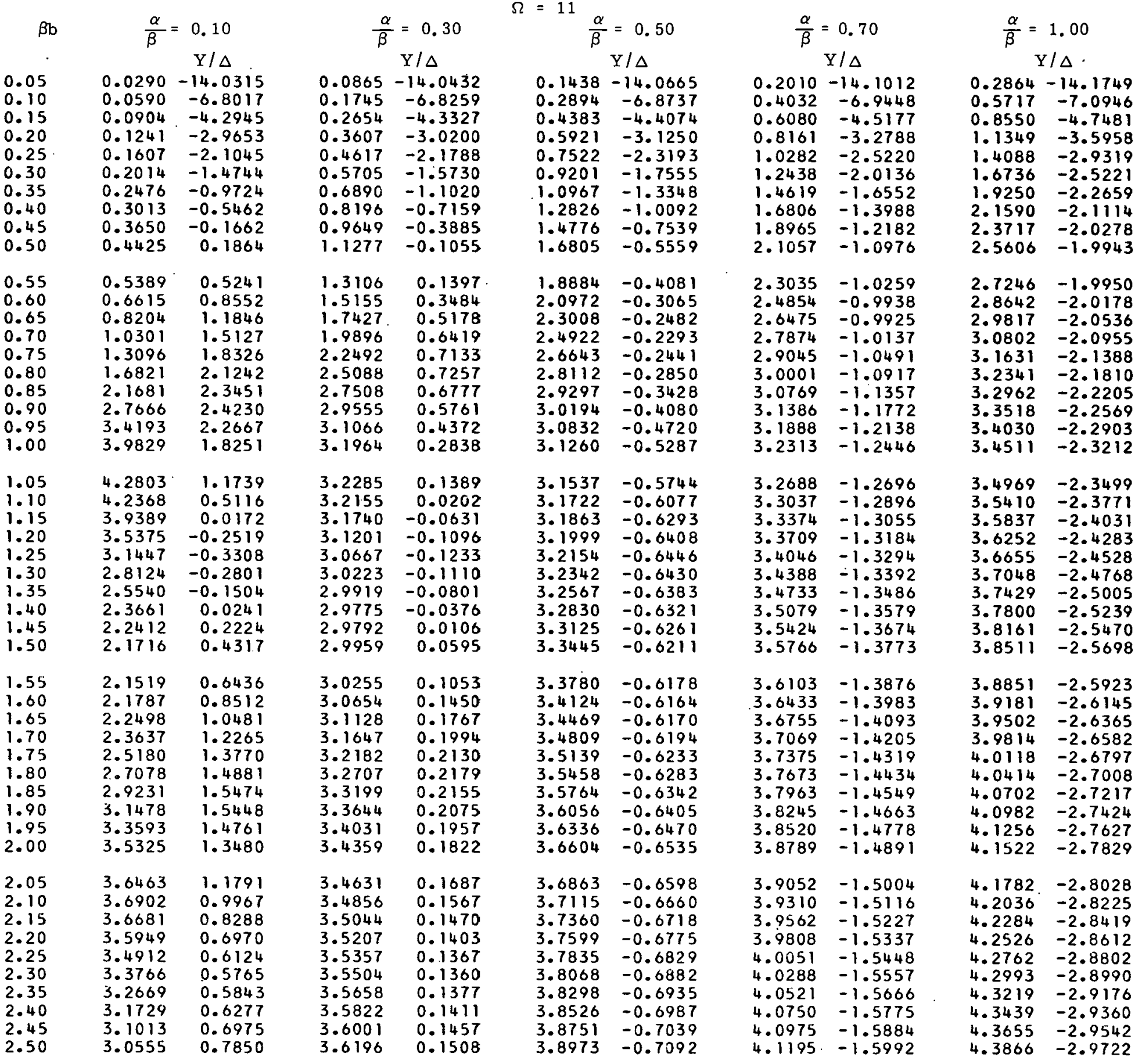


TABLE III

Normalized Admittance $\mathrm{Y} / \Delta$ of Loop Antennas in Dissipative Media

$\beta \mathrm{b}$

$$
\frac{\alpha}{\beta}=0.00
$$$$
\mathrm{Y} ! \Delta
$$

0.05

0.10

0.15

0.20

0.25

0.35

0.40

0.45

0.50

0.55

0.60

0.70

0.75
0.75

0.80

0.85
0.90

0.90
0.95

1.00

1.05

1.10
1.15

1.15
1.20

1.25

1.30
1.35

1.35

1.45

1.45
1.50

1.55
1.60

1.65

1.65

1.75

1.80
1.85

1.85
1.90

1.95

2.00

2.05

2.10
2.15

2.15
2.20

2.25

2.30

2.35

2.40

2.45

2.50
$0.0002-12.3838$

$0.0008-6.0079$

$0.0019-3.7985$

$0.0036-2.6282$

$0.0060-1.8703$

$0.0095-1.3150$

$0.0146-0.8711$

$0.0218-0.4917$

$0.0323-0.1495$

$0.0474 \quad 0.1742$

$\begin{array}{ll}0.0698 & 0.4934 \\ 0.1033 & 0.8207\end{array}$

$0.1544 \quad 1.1692$

$0.2347 \quad 1.5538$

$0.3646 \quad 1.9931$

0.58332 .5099

0.96933 .1257

$1.6821 \quad 3.8294$

$3.0140 \quad 4.4372$

$0.9797 \quad 1.9691$

$6.3872-0.7830$

$4.6500-1.9270$

$3.2716-1.9702$

$2.3889-1.6551$

$1.8314-1.2655$

$1.4688-0.8811$

$1.2260-0.5180$

$\begin{array}{lll}1.0616 & -0.1741\end{array}$

$0.9530 \quad 0.157 .8$

$\begin{array}{ll}0.8886 & 0.4858 \\ 0.8642 & 0.8179\end{array}$

$0.8814 \quad 1.1624$

$0.8814 \quad 1.1624$

$1.0832 \quad 1.9184$

$1.3152 \quad 2.3383$

$1.6944 \quad 2.7739$

$2.2933 \quad 3.1723$

$\begin{array}{ll}3.1836 & 3.3899\end{array}$

$5.2921 \quad 2.1830$

$5.5121,0.7726$

$4.9312-0.3661$

$4.0586-0.9108$

$3.2688-1.0093$

$2.6622-0.8742$

$2.2212-0.6356$

$1.9063-0.3555$

$1.6846-0.0599$

1.5338

0.2419

$\Omega=12$

$$
\begin{gathered}
\frac{\alpha}{\beta}=0.01 \\
\mathrm{Y} / \Delta
\end{gathered}
$$

$0.0026-12.3839$

$0.0057-6.0079$

$0.0094-3.7986$

$0.0137-2.6284$

$0.0191-1.8706$

$0.0258-1.3154$

$\begin{array}{lll}0.0343 & -0.8717\end{array}$

$0.0455-0.4928$

$0.0605-0.1511$

0.08110 .1716

$0.1100 \quad 0.4893$

$0.1516 \quad 0.8142$

$0.2131 \quad 1.1587$

$0.3068 \quad 1.5363$

$0.4544 \quad 1.9631$

0.69612 .4564

$1.1088 \quad 3.0265$

$1.8378 \quad 3.6393$

$3.1124 \quad 4.0862$

$4.9896 \quad 3.7064$

$6.4182 \quad 1.7662$

$5.9627-0.5402$

$4.5259-1.6197$

$3.2919-1.7477$

$2.4583-1.5089$

$1.9157-1.1693$

$1.5575-0.8168$

$1.3170-0.4750$

$1.1555-0.1464$

1.05140 .1730

$0.9936 \quad 0.4894$

$0.9780 \quad 0.8090$

$1.0067 \quad 1.1381$

$1.0880 \quad 1.4818$

$1.2381 \quad 1.8427$

$1.4838 \quad 2.2166$

$1.8656 \quad 2.5823$

$2.4329 \quad 2.8810$

$3.2132 \quad 2.9853$

$4.1192 \quad 2.6969$

$4.8422 \quad 1.8938$

$5.0070 \quad 0.8014$

$4.5938-0.1131$

$3.9217-0.6096$

$\begin{array}{ll}3.2661 & -0.7494\end{array}$

$2.7322-0.6745$

$2.3283-0.4892$

$\begin{array}{ll}2.0330 & -0.2514 \\ 1.8233 & 0.0107\end{array}$

$\begin{array}{ll}1.6816 & 0.2847\end{array}$ $\frac{\alpha}{\beta}=0.03$

$Y / \Delta$

$0.0075-12.3840$

$0.0156-6.0082$

$0.0244-3.7991$

$0.0341-2.6291$

$0.0452-1.8716$

$0.0581-1.3169$

$0.0736-0.8739$

$0.0926-0.4960$

$0.1167-0.1558$

0.14790 .1640

$0.1896 \quad 0.4788$

$0.2470 \quad 0.7980$

$0.3283 \quad 1.1332$

$0.4470 \quad 1.4951$

$0.6262 \quad 1.8945$

0.90602 .3385

$1.3561 \quad 2.8175$

$2.0877 \quad 3.2657$

$\begin{array}{ll}3.2198 & 3.4637 \\ 4.6349 & 2.9477\end{array}$

2.9477

$5.5604 \quad 1.4470$

$5.2650-0.2246$

$4.2584-1.1427$

$3.2807-1.3632$

$2.5554-1.2396$

$2.0552-0.9858$

$1.7149-0.6922$

$1.4842-0.3920$

$1.3314-0.0948$

1.2373

0.1983

1.1921

1.1922

1.2395

0.4896

0.7821

. 6079

3.1923

3.7835

2.3471

4.2051

4.3002

4.0706

3.6575

3.2090

2.8088

2.4846

2.2373
2.0584

1.9391 $\frac{\alpha}{\beta}=0.05$

$Y / \Delta$

$0.0124-12.3842$

$0.0254-6.0086$

$0.0393-3.7998$

$0.0545-2.6302$

$0.0713-1.8732$

$0.0905-1.3192$

$0.1128-0.8771$

$0.1396-0.5005$

$0.1726-0.1622$

0.21420 .1554

$\begin{array}{ll}0.2683 & 0.4652 \\ 0.3407 & 0.7777\end{array}$

$0.4403 \quad 1.1020$

$0.5814 \quad 1.4463$

$0.7871 \quad 1.8161$

$1.0952 \quad 2.2094$

$1.5639 \quad 2.6016$

$2.2692 \quad 2.9111$

$3.2489,2.9404$

$4.9408 \therefore 1.2056$

$4.7283-0.0478$

$3.9992-0.8072$

$3.2274-1.0562$

$2.6099-1.0065$

$2.1616-0.8194$

$1.8473-0.5770$

$1.6321-0.3156$

$1.6321-0.3156$

1.40790 .2164

$1.3745 \quad 0.4812$

$\begin{array}{ll}1.3873 & 0.7450\end{array}$

$\begin{array}{ll}1.4476 & 1.0071\end{array}$

$1.5605 \quad 1.2645$

$1.7343 \quad 1.5096$

$1.9788 \quad 1.7271$

$2.3013 \quad 1.8899$

$2.6959 \quad 1.9569$

$3.1289 \quad 1.8794$

$3.7880 \quad 1.2175$

$3.8449 \quad 0.7482$

$3.7043 \quad 0.3340$

$3.4383 \quad 0.0500$

$3.1297-0.0900$

$2.8363-0.1105$

$2.5863-0.0458$

$2.3889 \quad 0.0746$

$2.2442 \quad 0.2295$

$2.1489 \quad 0.4047$

$\frac{\alpha}{\beta}=0.07$ $\mathrm{Y} / \Delta$

$0.0173-12.3845$

$0.0353-6.0093$

$\begin{array}{lll}0.0543 & -3.8009\end{array}$

$0.0748-2.6317$

$0.0973-1.8754$

$0.1227-1.3222$

$0.1519-0.8813$

$0.1864-0.5063$

$0.2281-0.1704$

0.27990 .1438

$0.3459 \quad 0.4486$

$\begin{array}{ll}0.4324 & 0.7534\end{array}$

$0.5489 \quad 1.0657$

$0.7096 \quad 1.3909$

$0.9369-1.7297$

$1.2640 \quad 2.0727$

$1.7363 \quad 2.3854$

2.39772 .5825

$3.2328 \quad 2.5022$

$4.0503 \quad 1.9646$

$\begin{array}{lll}4.4768 & 1.0151\end{array}$

$\begin{array}{ll}4.3118 & 0.0497\end{array}$

$3.7666-0.5726$

$3.1547-0.8170$

$2.6355-0.8115$

$2.2416-0.6740$

$1.7618-0.2482$

$1.6344-0.0119$ 
TABLE III

Normalized Admittance $\mathrm{Y} / \Delta$ of Loop Antennas in Dissipative Media

$\beta 1$

1.05
0.10
0.15
0.20
0.25
0.30
0.35
0.40
0.45
0.50
0.55
0.60
0.65
0.70
0.75
0.80
0.85
0.90
0.95
1.00
1.05
1.10
1.15
1.20
1.25
1.30
1.35
1.40
1.45
1.50
1.55
1.60
1.65
1.70
1.75
1.80
1.85
1.90
1.95
2.00
2.05
2.10
2.15
2.20
2.25
2.30
2.35
2.40
2.45
2.50
0.40 $\frac{\alpha}{\beta}=0.10$

$0.0246-12.3851$

$0.0500-6.0107$

$0.0767-3.8031$

$0.1053-2.6349$

$0.1364-1.8799$

$0.1710-1.3283$

$0.2103-0.8895$

$0.2561-0.5175$

$0.3105-0.1858$

$0.3770 \quad 0.1224$

$0.4599 \quad 0.4184$

$0.5660 \quad 0.7100$

$0.7047 \quad 1.0025$

$\begin{array}{ll}0.7047 & 1.0025 \\ 0.8897 & 1.2971\end{array}$

$\begin{array}{ll}0.8897 & 1.2971 \\ 1.1403 & 1.5888\end{array}$

$1.4814 \quad 1.8602$

$1.9387 \quad 2.0703$

$2.5192 \quad 2.1430$

$3.1671 \quad 1.9724$

$3.7210 \quad 1.4915$

$3.9720 \quad 0.7912$

3.84790 .1131

$3.4768-0.3481$

$3.0383-0.5602$

$2.6421-0.5859$

$2.3258-0.4980$

$\begin{array}{ll}2.3258 & -0.4980 \\ 2.0906 & -0.3481\end{array}$

$\begin{array}{ll}2.0906 & -0.3481 \\ 1.9261 & -0.1669\end{array}$

1.82130 .0286

1.76730 .2287

$\begin{array}{ll}1.7583 & 0.4277\end{array}$

$\begin{array}{ll}1.7913 & 0.6207\end{array}$

$1.8649 \quad 0.8028$

$\begin{array}{ll}1.9786 & 0.9673\end{array}$

$2.1314 \quad 1.1049$

$2.3197 \quad 1.2040$

$2.5346 \quad 1.2506$

$2.7594 \quad 1.2325$

$2.9691 \quad 1.1438$

3.1348

0.9918

3.23230 .7986

$3.2512 \quad 0.5972$

$3.1994 \quad 0.4199$

$3.0976 \quad 0.2890$

$2.9705 \quad 0.2132$

0.8399000

.72120 .2109

$2.6238 \quad 0.2655$

$2.5523 \quad 0.3436$

$2.5088 \quad 0.4362$
$\Omega=12$

\begin{tabular}{|c|c|c|c|c|c|}
\hline \multicolumn{2}{|c|}{$\frac{\alpha}{\beta}=0.30$} & \multicolumn{2}{|c|}{$\frac{\alpha}{\beta}=0.50$} & \multicolumn{2}{|c|}{$\frac{\alpha}{\beta}=0.70$} \\
\hline $\begin{array}{l} \\
0.0734 \\
0.1482 \\
0.2256 \\
0.3066 \\
0.3929 \\
0.4860 \\
0.5877 \\
0.7003 \\
0.8262 \\
0.9680\end{array}$ & $\begin{array}{l}Y / \Delta \\
-12.3951 \\
-0.0312 \\
-3.8356 \\
-2.6814 \\
-1.9431 \\
-1.4125 \\
-1.0005 \\
-0.6632 \\
-0.3774 \\
-0.1304\end{array}$ & $\begin{array}{l}0.1221 \\
0.2458 \\
0.3725 \\
0.5035 \\
0.6403 \\
0.7840 \\
0.9356 \\
1.0958 \\
1.2644 \\
1.4405\end{array}$ & $\begin{array}{l}l \Delta \\
-12.4148 \\
-0.0718 \\
-3.8991 \\
-2.7709 \\
-2.0632 \\
-1.5689 \\
-1.2007 \\
-0.9165 \\
-0.6944 \\
-0.5232\end{array}$ & $\begin{array}{r}Y \\
0.1707 \\
0.3426 \\
0.5168 \\
0.6940 \\
0.8749 \\
1.0591 \\
1.2458 \\
1.4331 \\
1.6182 \\
1.7973\end{array}$ & $\begin{array}{l}\Delta \\
-12.4443 \\
-6.1323 \\
-3.9930 \\
-2.9021 \\
-2.2365 \\
-1.7901 \\
-1.4763 \\
-1.2528 \\
-1.0966 \\
-.0 .9940\end{array}$ \\
\hline $\begin{array}{l}1.1283 \\
1.3092 \\
1.5114 \\
1.7328 \\
1.9670 \\
2.2021 \\
2.4203 \\
2.6016 \\
2.7293 \\
2.7960\end{array}$ & $\begin{array}{l}0.0835 \\
0.2652 \\
0.4119 \\
0.5174 \\
0.5739 \\
0.5746 \\
0.5172 \\
0.4083 \\
0.2644 \\
0.1090\end{array}$ & $\begin{array}{l}1.6215 \\
1.8035 \\
1.9810 \\
2.1471 \\
2.2951 \\
2.4191 \\
2.5159 \\
2.5856 \\
2.6309 \\
2.6570\end{array}$ & $\begin{array}{l}-0.3969 \\
-0.3123 \\
-0.2670 \\
-0.2578 \\
-0.2796 \\
-0.3251 \\
-0.3855 \\
-0.4514 \\
-0.5149 \\
-0.5700\end{array}$ & $\begin{array}{l}1.9661 \\
2.1203 \\
2.2562 \\
2.3715 \\
2.4657 \\
2.5400 \\
2.5972 \\
2.6408 \\
2.6744 \\
2.7015\end{array}$ & $\begin{array}{l}-0.9351 \\
-0.9117 \\
-0.9161 \\
-0.9402 \\
-0.9767 \\
-1.0187 \\
-1.0612 \\
-1.1004 \\
-1.1343 \\
-1.1622\end{array}$ \\
\hline $\begin{array}{l}2.8065 \\
2.7747 \\
2.7184 \\
2.6537 \\
2.5928 \\
2.5435 \\
2.5095 \\
2.4921 \\
2.4909 \\
2.5043\end{array}$ & $\begin{array}{l}-0.0341 \\
-0.1480 \\
-0.2245 \\
-0.2641 \\
-0.2721 \\
-0.2559 \\
-0.2232 \\
-0.1809 \\
-0.1347 \\
-0.0892\end{array}$ & $\begin{array}{l}2.6699 \\
2.6752 \\
2.6776 \\
2.6806 \\
2.6864 \\
2.6960 \\
2.7098 \\
2.7275 \\
2.7484 \\
2.7717\end{array}$ & $\begin{array}{l}-0.6135 \\
-0.6445 \\
-0.6640 \\
-0.6740 \\
-0.6768 \\
-0.6749 \\
-0.6703 \\
-0.6649 \\
-0.6599 \\
-0.6563\end{array}$ & $\begin{array}{l}2.7248 \\
2.7463 \\
2.7673 \\
2.7887 \\
2.8107 \\
2.8334 \\
2.8567 \\
2.8803 \\
2.9039 \\
2.9273\end{array}$ & $\begin{array}{l}-1.1845 \\
-1.2019 \\
-1.2155 \\
-1.2264 \\
-1.2356 \\
-1.2439 \\
-1.2519 \\
-1.2600 \\
-1.2684 \\
-1.2772\end{array}$ \\
\hline $\begin{array}{l}2.5300 \\
2.5653 \\
2.6074 \\
2.6532 \\
2.6999 \\
2.7450 \\
2.7862 \\
2.8223 \\
2.8522 \\
2.8760\end{array}$ & $\begin{array}{r}-0.0479 \\
-0.0133 \\
0.0129 \\
0.0299 \\
0.0376 \\
0.0369 \\
0.0291 \\
0.0160 \\
-0.0002 \\
-0.0175\end{array}$ & $\begin{array}{l}2.7966 \\
2.8222 \\
2.8477 \\
2.8728 \\
2.8968 \\
2.9197 \\
2.9413 \\
2.9616 \\
2.9808 \\
2.9990\end{array}$ & $\begin{array}{l}-0.6545 \\
-0.6548 \\
-0.6570 \\
-0.6610 \\
-0.6665 \\
-0.6729 \\
-0.6800 \\
-0.6875 \\
-0.6950 \\
-0.7024\end{array}$ & $\begin{array}{l}2.9503 \\
2.9728 \\
2.9946 \\
3.0157 \\
3.0361 \\
3.0559 \\
3.0749 \\
3.0934 \\
3.1113 \\
3.1287\end{array}$ & $\begin{array}{l}-1.2865 \\
-1.2961 \\
-1.3061 \\
-1.3162 \\
-1.3265 \\
-1.3368 \\
-1.3472 \\
-1.3574 \\
-1.3676 \\
-1.3778\end{array}$ \\
\hline $\begin{array}{l}2.8943 \\
2.9079 \\
2.9181 \\
2.9263 \\
2.9336 \\
2.9410 \\
2.9495 \\
2.9594 \\
2.9711 \\
2.9845\end{array}$ & $\begin{array}{l}-0.0343 \\
-0.0490 \\
-0.0608 \\
-0.0692 \\
-0.0743 \\
-0.0765 \\
-0.0762 \\
-0.0742 \\
-0.0712 \\
-0.0679\end{array}$ & $\begin{array}{l}3.0163 \\
3.0330 \\
3.0492 \\
3.0651 \\
3.0807 \\
3.0961 \\
3.1113 \\
3.1264 \\
3.1413 \\
3.1561\end{array}$ & $\begin{array}{l}-0.7095 \\
-0.7164 \\
-0.7229 \\
-0.7291 \\
-0.7352 \\
-0.7410 \\
-0.7468 \\
-0.7525 \\
-0.7583 \\
-0.7641\end{array}$ & $\begin{array}{l}3.1457 \\
3.1622 \\
3.1783 \\
3.1940 \\
3.2094 \\
3.2244 \\
3.2390 \\
3.2534 \\
3.2674 \\
3.2811\end{array}$ & $\begin{array}{l}-1.3878 \\
-1.3977 \\
-1.4076 \\
-1.4174 \\
-1.4272 \\
-1.4369 \\
-1.4465 \\
-1.4561 \\
-1.4657 \\
-1.4753\end{array}$ \\
\hline
\end{tabular}

$$
\frac{\alpha}{\beta}=1.00
$$$$
\mathrm{Y} / \Delta
$$

$0.2433-12.5069$

$0.4857-6.2596$

$0.7265-4.189$

$0.9644-3.1724$

$1.1973-2.5867$

$1.4220-2.2256$

$\begin{array}{ll}1.4220 & -2.2256 \\ 1.6350 & -2.0003\end{array}$

$\begin{array}{ll}1.6350 & -2.0003 \\ 1.8323 & -1.8652\end{array}$

$2.0105-1.7931$

$2.1670-1.7652$

$2.3008-1.7675$

$2.4126-1.7890$

$2.5043-1.8214$

$2.5789-1.8585$

$2.6398-1.8962$

$2.6903-1.9322$

$2.7332-1.9653$

$2.7709-1.9953$

. $0051-2.0225$

$\begin{array}{ll}2.8051 & -2.0225 \\ 2.8369 & -2.0471\end{array}$

$2.867 .1-2.0699$

$2.8961-2.0913$

$2.9242-2.1117$

$.2 .9514 \quad-2.1313$

$2.9779-2.1505$

$3.0035-2.1693$

$3.0283-2.1878$

$3.0523-2.2060$

$3.0755-2.224$

$3.0979-2.2419$

$3.1195 \quad-2.2595$

$3.1403-2.2760$

$3.1604-2.2941$

$3.1798-2.3110$

$3.1986-2.3277$

$3.2167-2.3442$

$3.2343-2.3604$

$3.2513-2.3765$

$3.2677-2.3923$

$3.2836-2.4079$

$3.2990-2.4234$

$3.3139-2.4386$

$3.3284-2.4537$

$3.3424-2.4686$

$3.356 a-2.4833$

$3.3692-2.4979$

$3.3820-2.5123$

$3.3944-2.5265$

$3.4064-2.5406$

$3.4180-2.5545$ 
TABLE IV

Normalized Admittance $Y / \Delta$ of Loop Antennas in Dissipative Mecia

$\beta \mathrm{b} \quad \frac{\alpha}{\beta}=0.00$

$\mathrm{Y} / \Delta$

\begin{tabular}{|c|c|c|}
\hline $\begin{array}{l}0.05 \\
0.10 \\
0.15 \\
0.20 \\
0.25 \\
0.30 \\
0.35 \\
0.40 \\
0.45 \\
0.50\end{array}$ & $\begin{array}{l}0.0001 \\
0.0004 \\
0.0010 \\
0.0019 \\
0.0033 \\
0.0052 \\
0.0080 \\
0.0120 \\
0.0178 \\
0.0262\end{array}$ & $\begin{array}{r}-9.1554 \\
-4.4503 \\
-2.8236 \\
-1.9645 \\
-1.4101 \\
-1.0053 \\
-0.6827 \\
-0.4077 \\
-0.1597 \\
0.0751\end{array}$ \\
\hline $\begin{array}{l}0.55 \\
0.60 \\
0.65 \\
0.70 \\
0.75 \\
0.80 \\
0.85 \\
0.90 \\
0.95 \\
1.00\end{array}$ & $\begin{array}{l}0.0386 \\
0.0574 \\
0.0865 \\
0.1330 \\
0.2105 \\
0.3471 \\
0.0069 \\
1.1506 \\
2.4073 \\
5.1475\end{array}$ & $\begin{array}{l}0.3076 \\
0.5477 \\
0.8065 \\
1.0977 \\
1.4408 \\
1.8645 \\
2.4125 \\
3.1369 \\
3.9786 \\
3.9093\end{array}$ \\
\hline $\begin{array}{l}1.05 \\
1.10 \\
1.15 \\
1.20 \\
1.25 \\
1.30 \\
1.35 \\
1.40 \\
1.45 \\
1.50\end{array}$ & $\begin{array}{l}7.1521 \\
4.8807 \\
2.8139 \\
1.7662 \\
1.2225 \\
0.9134 \\
0.7239 \\
0.6016 \\
0.5214 \\
0.4702\end{array}$ & $\begin{array}{r}0.2902 \\
-2.5090 \\
-2.5757 \\
-2.0714 \\
-1.5815 \\
-1.1718 \\
-0.8280 \\
-0.5295 \\
-0.2599 \\
-0.0066\end{array}$ \\
\hline $\begin{array}{l}1.55 \\
1.60 \\
1.65 \\
1.70 \\
1.75 \\
1.80 \\
1.85 \\
1.90 \\
1.95 \\
2.00\end{array}$ & $\begin{array}{l}0.4422 \\
0.4354 \\
0.4520 \\
0.4988 \\
0.5901 \\
0.7540 \\
1.0470 \\
1.5822 \\
2.5642 \\
4.1394\end{array}$ & $\begin{array}{l}0.2409 \\
0.4918 \\
0.7555 \\
1.0428 \\
1.3663 \\
1.7408 \\
2.1790 \\
2.6677 \\
3.0700 \\
2.8425\end{array}$ \\
\hline $\begin{array}{l}2.05 \\
2.10 \\
2.15 \\
2.20 \\
2.25 \\
2.30 \\
2.35 \\
2.40 \\
2.45 \\
2.50\end{array}$ & $\begin{array}{l}5.4573 \\
4.9449 \\
3.5420 \\
2.4554 \\
1.7788 \\
1.3627 \\
1.0987 \\
0.9260 \\
0.8119 \\
0.7387\end{array}$ & $\begin{array}{r}1.1474 \\
-0.9671 \\
-1.8088 \\
-1.7836 \\
-1.4964 \\
-1.1686 \\
-0.8571 \\
-0.5702 \\
-0.3036 \\
-0.0499\end{array}$ \\
\hline
\end{tabular}

$$
\frac{\alpha}{\beta}=0.01
$$$$
\mathrm{Y} / \Delta
$$

$0.0018-9.1554$

$0.0038-4.4503$

$0.0062-2.8236$

$0.0090-1.9646$

$0.0124-1.4103$

$0.0165-1.0056$

$0.0218-0.6831$

$0.0286-0.4083$

$0.0377-0.1606$

0.05010 .0736

\section{$0.0674 \quad 0.3052$}

$0.0925 \quad 0.5439$

$0.1298 \quad 0.8003$

$0.1874 \quad 1.0872$

$0.4403 \quad 1.4295$

$0.7334 \quad 2.3416$

$\begin{array}{ll}0.7334 & 2.3416 \\ 1.3176 & 2.9793\end{array}$

$2.5577 \quad 3.6081$

$4.8714 \quad 3.2605$

$\begin{array}{lll}0.3132 & 0.3242\end{array}$

$4.6364-2.0294$

$2.8608-2.2810$

$1.8597-1.9144$

$1.3131-1.4928$

$0.9954-1.1182$

$0.7994-0.7940$

$\begin{array}{lll}0.6738 & -0.5077\end{array}$

$0.5930-0.2466$

$0.5439-0.0001$

$0.5203 \quad 0.2409$

$\begin{array}{ll}0.5209 & 0.4846 \\ 0.5481 & 0.7390\end{array}$

$\begin{array}{ll}0.5481 & 0.7390 \\ 0.6095 & 1.0125\end{array}$

$0.7201 \quad 1.3139$

$0.9083 \quad 1.6506$

$\begin{array}{ll}1.2257 & 2.0204\end{array}$

$1.7624 \quad 2.3840$

$2.6377 \quad 2.5848$

$3.8244 \quad 2.2215$

$4.6704 \quad 0.9285$

$4.3613-0.5862$

$3.3844-1.3482$

$2.5010-1.4552$

$1.8880-1.2847$

$1.4859-1.0324$

$1.2218-0.7680$

$\therefore .0466-0.5119$

$0.9312-0.2667$

$0.8592-0.0298$

\section{$\Omega=15$}

$\frac{\alpha}{\beta}=0.03$

$\mathrm{Y} / . \Delta$

$\begin{array}{ll}0.0052 & -9.1555 \\ 0.0107 & -4.4505\end{array}$

$0.0107-4.4505$

$0.0166-2.8239$

$0.0232-1.9651$

$0.0306-1.4109$

$0.0391-1.0065$

$0.0493-0.6345$

$0.0618-0.4703$

$0.0775-0.1635$

0.09770 .0693

$0.1247 \quad 0.2987$

$0.1619 \quad 0.5339$

$0.2150 \quad 0.7843$

$0.2937 \quad 1.0609$

$0.4158 \quad 1.3769$

$0.6149 \quad 1.7474$

0.95882 .1830

$\begin{array}{ll}1.5834 & 2.6539\end{array}$

$2.7152 \quad 2.9469$

$4.3462 \quad 2.3426$

$\begin{array}{ll}5.1358 & 0.3176\end{array}$

$\begin{array}{lll}4.1444 & -1.3637\end{array}$

$2.8541-1.7777$

$1.9844-1.6150$

$\begin{array}{ll}1.4598 & -1.3141 \\ 1.1394 & -1.0073\end{array}$

$0.9380-0.7234$

$0.8094-0.4632$

$0.7293-0.2210$

$0.6848 \quad 0.0099$

0.66990 .2358

0.6835

0.7283

0.8123

0.9497

1.1639

1.4899

1.9692
2.6124
3.2960

3.2960

0.4623

0.6947

0.8369

1.1905

1.4509
1.6 .972

1.8714

1.8484
1.4524

$3.6910 \quad 0.6556$

$3.5472-0.2063$

$3.0341-0.7565$

$2.4674-0.9489$

$2.0019-0.9170$

$1.6593-0.7778$

$\begin{array}{lll}1.4176 & -0.5948\end{array}$

$1.2515-0.3969$

$1.1417-0.1956$

$\begin{array}{ll}1.0759 & 0.0053\end{array}$

\begin{tabular}{|c|c|}
\hline \multicolumn{2}{|c|}{$\begin{array}{c}\frac{\alpha}{\beta}=0.05 \\
Y: \Delta\end{array}$} \\
\hline $\begin{array}{l}.0086 \\
.0175 \\
.0270 \\
.0373 \\
.0487 \\
.0617 \\
.0768 \\
.0949 \\
.1170 \\
.1450\end{array}$ & $\begin{array}{r}-9.1556 \\
-4.4508 \\
-2.8244 \\
-1.9658 \\
-1.4120 \\
-1.0080 \\
-0.0866 \\
-0.4132 \\
-0.1677 \\
0.0633\end{array}$ \\
\hline $\begin{array}{l}1814 \\
2302 \\
2981 \\
3960 \\
5429 \\
.7729 \\
1478 \\
7708 \\
7497 \\
9015\end{array}$ & $\begin{array}{l}0.2899 \\
0.5206 \\
0.7637 \\
1.0280 \\
1.3221 \\
1.0523 \\
2.0103 \\
2.3338 \\
2.4001 \\
1.7417\end{array}$ \\
\hline $\begin{array}{l}.3563 \\
.7130 \\
.7712 \\
.0448 \\
.5655 \\
.2576 \\
.0592 \\
.9325 \\
.8555 \\
.8163\end{array}$ & $\begin{array}{r}0.2758 \\
-0.9550 \\
-1.3890 \\
-1.3498 \\
-1.1434 \\
-0.8973 \\
-0.6526 \\
-0.4194 \\
-0.1977 \\
0.0156\end{array}$ \\
\hline $\begin{array}{l}.8092 \\
.8332 \\
8909 \\
.9889 \\
1384 \\
3551 \\
.6560 \\
0488 \\
5029 \\
9132\end{array}$ & $\begin{array}{l}0.2241 \\
0.4310 \\
0.6387 \\
0.8473 \\
1.0532 \\
1.2448 \\
1.3957 \\
1.4550 \\
1.3478 \\
1.0125\end{array}$ \\
\hline $\begin{array}{l}.1192 \\
.0348 \\
.7364 \\
.3691 \\
.0317 \\
.7602 \\
.5564 \\
.4112 \\
.3145 \\
.2585\end{array}$ & $\begin{array}{r}0.4895 \\
-0.0492 \\
-0.4326 \\
-0.0141 \\
-0.6384 \\
-0.5666 \\
-0.4432 \\
-0.2944 \\
-0.1340 \\
0.0311\end{array}$ \\
\hline
\end{tabular}

$\frac{\alpha}{\beta}=0.07$

$\mathrm{Y} / \Delta$

$0.0119-9.1558$

$0.0243-4.4512$

$0.0374-2.8251$

$\begin{array}{lll}0.0514 & -1.9669\end{array}$

$0.0669-1.4135$

$0.0842-1.0101$

$0.1042-0.6894$

$0.1278-0.4171$

$0.1563-0.1732$

$\begin{array}{lll}0.1918 & 0.0556\end{array}$

0.23730 .2787

$0.2972 \quad 0.5041$

$0.3789 \quad 0.7388$

$0.4938 \quad 0.9891$

$0.6614 \quad 1.2594$

$0.9137 \quad 1.5476$

$1.3024 \quad 1.8316$

$1.8960 \quad 2.0330$

$2.7177 \quad 1.9565$

$3.5390 \quad 1.3264$

$\begin{array}{ll}3.8075 & 0.2255\end{array}$

$3.3577-0.6988$

$2.6579-1.0977$

$2.0621-1.1262$

$\begin{array}{lll}1.6381 & -0.9888\end{array}$

$1.3524-0.7937$

$1.1638-0.5850$

$\begin{array}{ll}1.0427 & -0.3783\end{array}$

$0.9707-0.1777$

$\begin{array}{ll}0.9372 & 0.0169\end{array}$

$0.9371 \quad 0.2067$

$\begin{array}{lll}0.9690 & 0.3927\end{array}$

$\begin{array}{lll}1.0348 & 0.5747\end{array}$

$\begin{array}{ll}1.1394 & 0.7503\end{array}$

$1.2898 \quad 0.9126$

$1.4937 \quad 1.0479$

$1.7552 \quad 1.1318$

$2.0643 \quad 1.1287$

2.38331 .0005

$2.6400 \quad 0.7342$

$2.7553 \quad 0.3745$

$2.0983 \quad 0.0167$

$2.5100-0.2522$

$2.2650-0.3999$

$2.0233-0.4406$

$1.8160-0.4052$

$1.6527-0.3222$

$1.5330-0.2116$

$1.4528-0.0861$

1.40810 .0461 
TABLE IV

Normalized Admittance $\mathrm{Y} / \Delta$ of Loop Antennas in Dissipative Media

$\Omega=15$

\begin{tabular}{|c|c|c|c|c|}
\hline$\beta b$ & $\frac{\alpha}{\beta}=$ & 0.10 & $\frac{\alpha}{\beta}=$ & 0.30 \\
\hline $\begin{array}{l}05 \\
10 \\
15 \\
20 \\
25 \\
30 \\
35\end{array}$ & $\begin{array}{l}0.0170 \\
0.0346 \\
0.0529 \\
0.0726 \\
0.0940 \\
0.1179 \\
0.1451 \\
0.1769 \\
0.2148 \\
0.2612\end{array}$ & $\begin{array}{l}Y / \Delta \\
-9.1563 \\
-4.4522 \\
-2.8266 \\
-1.9690 \\
-1.4165 \\
-1.0142 \\
-0.6950 \\
-0.4248 \\
-0.1837 \\
0.0409\end{array}$ & $\begin{array}{l}0.0508 \\
0.1026 \\
0.1562 \\
0.2126 \\
0.2728 \\
0.3380 \\
0.4098 \\
0.4898 \\
0.5801 \\
0.6829\end{array}$ & $\begin{array}{l}Y / \Delta \\
-9.1632 \\
-4.4664 \\
-2.8491 \\
-2.0012 \\
-1.4604 \\
-1.0728 \\
-0.7726 \\
-0.5273 \\
-0.3194 \\
-0.1398\end{array}$ \\
\hline 00 & $\begin{array}{l}0.3195 \\
0.3949 \\
0.4949 \\
0.6314 \\
0.8221 \\
1.0933 \\
1.4784 \\
2.0007 \\
2.6147 \\
3.11199\end{array}$ & $\begin{array}{l}0.2579 \\
0.4740 \\
0.6941 \\
0.9212 \\
1.1537 \\
1.3800 \\
1.5656 \\
1.6315 \\
1.4445 \\
0.9046\end{array}$ & $\begin{array}{l}0.8006 \\
0.9352 \\
1.0880 \\
1.2580 \\
1.4403 \\
1.6242 \\
1.7931 \\
1.9274 \\
2.0108 \\
2.0375\end{array}$ & $\begin{array}{r}0.0158 \\
0.1478 \\
0.2532 \\
0.3263 \\
0.3592 \\
0.3444 \\
0.2788 \\
0.1682 \\
0.0294 \\
-0.1139\end{array}$ \\
\hline $\begin{array}{l}45 \\
50\end{array}$ & $\begin{array}{l}3.2410 \\
2.9489 \\
2.4815 \\
2.0429 \\
1.7024 \\
1.4588 \\
1.2923 \\
1.1848 \\
1.1229 \\
1.0983\end{array}$ & $\begin{array}{r}0.1508 \\
-0.4763 \\
-0.7973 \\
-0.8667 \\
-0.7948 \\
-0.6578 \\
-0.4951 \\
-0.3251 \\
-0.1554 \\
0.0107\end{array}$ & $\begin{array}{l}2.0148 \\
1.9589 \\
1.8878 \\
1.8163 \\
1.7540 \\
1.7059 \\
1.6737 \\
1.6572 \\
1.6550 \\
1.6651\end{array}$ & $\begin{array}{l}-0.2388 \\
-0.3309 \\
-0.3859 \\
-0.4074 \\
-0.4024 \\
-0.3792 \\
-0.3447 \\
-0.3050 \\
-0.2645 \\
-0.2268\end{array}$ \\
\hline & $\begin{array}{l}1.1065 \\
1.1458 \\
1.2166 \\
1.3203 \\
1.4586 \\
1.0306 \\
1.8298 \\
2.0394 \\
2.2303 \\
2.3663\end{array}$ & $\begin{array}{l}0.1716 \\
0.3258 \\
0.4707 \\
0.6016 \\
0.7104 \\
0.7851 \\
0.8097 \\
0.7681 \\
0.6512 \\
0.4680\end{array}$ & $\begin{array}{l}1.6852 \\
1.7129 \\
1.7453 \\
1.7800 \\
1.8143 \\
1.8459 \\
1.8731 \\
1.8948 \\
1.9104 \\
1.9203\end{array}$ & $\begin{array}{l}-0.1944 \\
-0.1692 \\
-0.1522 \\
-0.1439 \\
-0.1439 \\
-0.1513 \\
-0.1645 \\
-0.1817 \\
-0.2007 \\
-0.2197\end{array}$ \\
\hline 05 & $\begin{array}{l}2.4187 \\
2.3825 \\
2.2778 \\
2.1369 \\
1.9897 \\
1.8558 \\
1.7454 \\
1.6623 \\
1.6070 \\
1.5782\end{array}$ & $\begin{array}{r}0.2503 \\
0.0418 \\
-0.1205 \\
-0.2198 \\
-0.2578 \\
-0.2461 \\
-0.1988 \\
-0.1280 \\
-0.0433 \\
0.0482\end{array}$ & $\begin{array}{l}1.9253 \\
1.9265 \\
1.9252 \\
1.9228 \\
1.9204 \\
1.9189 \\
1.9189 \\
1.9209 \\
1.9247 \\
1.9304\end{array}$ & $\begin{array}{l}-0.2371 \\
-0.2516 \\
-0.2627 \\
-0.2703 \\
-0.2746 \\
-0.2762 \\
-0.2756 \\
-0.2738 \\
-0.2714 \\
-0.2691\end{array}$ \\
\hline
\end{tabular}

\begin{tabular}{|c|c|c|c|}
\hline \multicolumn{2}{|c|}{$\frac{\alpha}{\beta}=0.50$} & \multicolumn{2}{|c|}{$\frac{\alpha}{\beta}=0.70$} \\
\hline $\begin{array}{l}0.0845 \\
0.1702 \\
0.2581 \\
0.3494 \\
0.4449 \\
0.5458 \\
0.6529 \\
0.7669 \\
0.8876 \\
1.0146\end{array}$ & $\begin{array}{l}-9.1768 \\
-4.4945 \\
-2.8932 \\
-2.0635 \\
-1.5443 \\
-1.1828 \\
-0.9143 \\
-0.7078 \\
-0.5475 \\
-0.4252\end{array}$ & $\begin{array}{l}0.1182 \\
0.2373 \\
0.3582 \\
0.4815 \\
0.6077 \\
0.7367 \\
0.8678 \\
0.9997 \\
1.1302 \\
1.2562\end{array}$ & $\begin{array}{l}-9.1972 \\
-4.5363 \\
-2.9584 \\
-2.1549 \\
-1.6657 \\
-1.3386 \\
-1.1096 \\
-0.9477 \\
-0.8363 \\
-0.7654\end{array}$ \\
\hline $\begin{array}{l}1.1460 \\
1.2785 \\
1.4076 \\
1.5273 \\
1.6316 \\
1.7154 \\
1.7758 \\
1.8133 \\
1.8308 \\
1.8335\end{array}$ & $\begin{array}{l}-0.3370 \\
-0.2811 \\
-0.2560 \\
-0.2596 \\
-0.2879 \\
-0.3347 \\
-0.3920 \\
-0.4517 \\
-0.5068 \\
-0.5526\end{array}$ & $\begin{array}{l}1.3742 \\
1.4804 \\
1.5718 \\
1.0464 \\
1.7038 \\
1.7453 \\
1.7734 \\
1.7913 \\
1.8022 \\
1.8090\end{array}$ & $\begin{array}{l}-0.7277 \\
-0.7171 \\
-0.7276 \\
-0.7527 \\
-0.7862 \\
-0.8227 \\
-0.8579 \\
-0.8891 \\
-0.9149 \\
-0.9351\end{array}$ \\
\hline $\begin{array}{l}1.8268 \\
1.8159 \\
1.8046 \\
1.7956 \\
1.7904 \\
1.7894 \\
1.7926 \\
1.7995 \\
1.8092 \\
1.8210\end{array}$ & $\begin{array}{l}-0.5869 \\
-0.6097 \\
-0.6224 \\
-0.6273 \\
-0.6267 \\
-0.6228 \\
-0.6175 \\
-0.6123 \\
-0.6080 \\
-0.6054\end{array}$ & $\begin{array}{l}1.8139 \\
1.8184 \\
1.8235 \\
1.8296 \\
1.8368 \\
1.8450 \\
1.8540 \\
1.8634 \\
1.8730 \\
1.8826\end{array}$ & $\begin{array}{l}-0.9503 \\
-0.9613 \\
-0.9694 \\
-0.9755 \\
-0.9805 \\
-0.9850 \\
-0.9895 \\
-0.9942 \\
-0.9994 \\
-1.0051\end{array}$ \\
\hline $\begin{array}{l}1.8340 \\
1.8473 \\
1.8605 \\
1.8731 \\
1.8847 \\
1.8952 \\
1.9046 \\
1.9130 \\
1.9204 \\
1.9272\end{array}$ & $\begin{array}{l}-0.6047 \\
-0.6059 \\
-0.6089 \\
-0.6134 \\
-0.6190 \\
-0.6254 \\
-0.6321 \\
-0.6389 \\
-0.6456 \\
-0.6520\end{array}$ & $\begin{array}{l}1.8919 \\
1.9009 \\
1.9094 \\
1.9175 \\
1.9251 \\
1.9322 \\
1.9389 \\
1.9453 \\
1.9514 \\
1.9572\end{array}$ & $\begin{array}{l}-1.0112 \\
-1.0176 \\
-1.0242 \\
-1.0310 \\
-1.0379 \\
-1.0447 \\
-1.0515 \\
-1.0582 \\
-1.0649 \\
-1.0714\end{array}$ \\
\hline $\begin{array}{l}1.9333 \\
1.9391 \\
1.9447 \\
1.9501 \\
1.9555 \\
1.9609 \\
1.9663 \\
1.9716 \\
1.9770 \\
1.9823\end{array}$ & $\begin{array}{l}-0.6580 \\
-0.6637 \\
-0.6690 \\
-0.6740 \\
-0.6787 \\
-0.6833 \\
-0.6879 \\
-0.6924 \\
-0.6969 \\
-0.7015\end{array}$ & $\begin{array}{l}1.9627 \\
1.9680 \\
1.9731 \\
1.9780 \\
1.9827 \\
1.9873 \\
1.9917 \\
1.9959 \\
2.0000 \\
2.0039\end{array}$ & $\begin{array}{l}-1.0778 \\
-1.0842 \\
-1.0905 \\
-1.0967 \\
-1.1028 \\
-1.1089 \\
-1.1150 \\
-1.1210 \\
-1.1270 \\
-1.1330\end{array}$ \\
\hline
\end{tabular}

\begin{tabular}{|c|c|}
\hline \multicolumn{2}{|c|}{$\frac{\alpha}{\beta}=1.00$} \\
\hline $\begin{array}{l}0.1685 \\
0.3364 \\
0.5033 \\
0.6684 \\
0.8298 \\
0.9854 \\
1.1322 \\
1.2671 \\
1.3871 \\
1.4903\end{array}$ & $\begin{array}{l}/ \Delta \\
-9.2406 \\
-4.0246 \\
-3.0947 \\
-2.3434 \\
-1.9108 \\
-1.0446 \\
-1.4793 \\
-1.3812 \\
-1.3300 \\
-1.3118\end{array}$ \\
\hline $\begin{array}{l}1.5756 \\
1.6438 \\
1.6965 \\
1.7362 \\
1.7659 \\
1.7882 \\
1.8056 \\
1.8197 \\
1.8319 \\
1.8431\end{array}$ & $\begin{array}{l}-1.3157 \\
-1.3333 \\
-1.3579 \\
-1.3849 \\
-1.41114 \\
-1.4355 \\
-1.4568 \\
-1.4753 \\
-1.4913 \\
-1.5053\end{array}$ \\
\hline $\begin{array}{l}1.8536 \\
1.8637 \\
1.8736 \\
1.8832 \\
1.8925 \\
1.9015 \\
1.9100 \\
1.9182 \\
1.9259 \\
1.9332\end{array}$ & $\begin{array}{l}-1.5178 \\
-1.5294 \\
-1.5402 \\
-1.5507 \\
-1.5608 \\
-1.5708 \\
-1.5806 \\
-1.5904 \\
-1.6000 \\
-1.6095\end{array}$ \\
\hline $\begin{array}{l}1.9400 \\
1.9465 \\
1.9525 \\
1.9582 \\
1.9636 \\
1.9686 \\
1.9733 \\
1.9778 \\
1.9819 \\
1.9858\end{array}$ & $\begin{array}{l}-1.6188 \\
-1.6280 \\
-1.6371 \\
-1.6460 \\
-1.6547 \\
-1.6634 \\
-1.6718 \\
-1.6802 \\
-1.6883 \\
-1.6964\end{array}$ \\
\hline $\begin{array}{l}1.9894 \\
1.9928 \\
1.9960 \\
1.9989 \\
2.0016 \\
2.0041 \\
2.0064 \\
2.0086 \\
2.0105\end{array}$ & $\begin{array}{l}-1.7043 \\
-1.7122 \\
-1.7199 \\
-1.7274 \\
-1.7349 \\
-1.7423 \\
-1.7496 \\
-1.7567 \\
-1.7638 \\
-1.7708\end{array}$ \\
\hline
\end{tabular}


TABLE V

Normalized Admittance $Y / \Delta$ of Loop Antennas in Dissipative Media

$\beta \mathrm{b}$

$\mathrm{Y} / \Delta$

0.05

0.10

0.15

0.20

0.25

0.30

0.45

0.50

0.55

0.60

0.65

0.75

0.80

0.85

0.90

0.95

1.00

1.05

1.10

1.15

1.20

1.25

1.30

1.35

1.45

1.50

1.55

1.60

1.65

1.70

1.75

1.80

1.85

1.90

1.95

2.00

2.05

2.10

2.20

2.25

2.30

2.35

2.40

2.45

2.50

$$
\frac{\alpha}{\beta}=0.00
$$

$\Omega=17$

\begin{tabular}{|c|c|c|c|}
\hline \multicolumn{2}{|c|}{$\mathrm{Y} / \Delta$} & \multicolumn{2}{|c|}{$Y / \Delta$} \\
\hline $\begin{array}{l}0.0001 \\
0.0003 \\
0.0007 \\
0.0013 \\
0.0022 \\
0.0035 \\
0.0054 \\
0.0081 \\
0.0120 \\
0.0177\end{array}$ & $\begin{array}{r}-7.5205 \\
-3.6585 \\
-2.3246 \\
-1.6211 \\
-1.1677 \\
-0.8372 \\
-0.5741 \\
-0.3500 \\
-0.1479 \\
0.0435\end{array}$ & $\begin{array}{l}0.0014 \\
0.0030 \\
0.0048 \\
0.0070 \\
0.0095 \\
0.0126 \\
0.0165 \\
0.0215 \\
0.0281 \\
0.0371\end{array}$ & $\begin{array}{r}-7.5205 \\
-3.6586 \\
-2.3247 \\
-1.6211 \\
-1.1678 \\
-0.8373 \\
-0.5744 \\
-0.3504 \\
-0.1486 \\
0.0424\end{array}$ \\
\hline $\begin{array}{l}0.0262 \\
0.0391 \\
0.0591 \\
0.0913 \\
0.1459 \\
0.2444 \\
0.4389 \\
0.8742 \\
2.0206 \\
5.1373\end{array}$ & $\begin{array}{l}0.2333 \\
0.4302 \\
0.6436 \\
0.8859 \\
1.1755 \\
1.5418 \\
2.0347 \\
2.7367 \\
3.6945 \\
3.8032\end{array}$ & $\begin{array}{l}0.0496 \\
0.0677 \\
0.0947 \\
0.1366 \\
0.2053 \\
0.3254 \\
0.5542 \\
1.0412 \\
2.2073 \\
4.7883\end{array}$ & $\begin{array}{l}0.2317 \\
0.4275 \\
0.6392 \\
0.8784 \\
1.1621 \\
1.5158 \\
1.9795 \\
2.6034 \\
3.3276 \\
3.0269\end{array}$ \\
\hline $\begin{array}{l}0.9020 \\
3.6382 \\
1.8759 \\
1.1348 \\
0.7756 \\
0.5773 \\
0.4575 \\
0.3810 \\
0.3313 \\
0.3002\end{array}$ & $\begin{array}{l}-0.9520 \\
-2.9701 \\
-2.4507 \\
-1.8366 \\
-1.3653 \\
-1.0030 \\
-0.7108 \\
-0.4625 \\
-0.2407 \\
-0.0334\end{array}$ & $\begin{array}{l}5.9742 \\
3.5808 \\
1.9839 \\
1.2374 \\
0.8601 \\
0.6487 \\
0.5211 \\
0.4406 \\
0.3899 \\
0.3603\end{array}$ & $\begin{array}{l}-0.6197 \\
-2.4677 \\
-2.2219 \\
-1.7272 \\
-1.3063 \\
-0.9682 \\
-0.6892 \\
-0.4488 \\
-0.2326 \\
-0.0298\end{array}$ \\
\hline $\begin{array}{l}0.2840 \\
0.2817 \\
0.2953 \\
0.3301 \\
0.3976 \\
0.5216 \\
0.7542 \\
1.2167 \\
2.1930 \\
4.0749\end{array}$ & $\begin{array}{l}0.1691 \\
0.3752 \\
0.5939 \\
0.8357 \\
1.1146 \\
1.4501 \\
1.8676 \\
2.3858 \\
2.9156 \\
2.7502\end{array}$ & $\begin{array}{l}0.3479 \\
0.3521 \\
0.3753 \\
0.4239 \\
0.5111 \\
0.6624 \\
0.9297 \\
1.4178 \\
2.3105 \\
3.6688\end{array}$ & $\begin{array}{l}0.1684 \\
0.3695 \\
0.5814 \\
0.8130 \\
1.0749 \\
1.3791 \\
1.7349 \\
2.1237 \\
2.3957 \\
1.9994\end{array}$ \\
\hline $\begin{array}{l}5.5169 \\
4.1584 \\
2.5313 \\
1.6193 . \\
1.1321 \\
0.8541 \\
0.6847 \\
0.5766 \\
0.5066 \\
0.4627\end{array}$ & $\begin{array}{r}0.3773 \\
-1.8458 \\
-2.1189 \\
-1.7831 . \\
-1.3982 \\
-1.0616 \\
-0.7746 \\
-0.5244 \\
-0.2986 \\
-0.0871\end{array}$ & $\begin{array}{l}4.4957 \\
3.7161 \\
2.5417 \\
1.7409 \\
1.2637 \\
0.9759 \\
0.7960 \\
0.6806 \\
0.6070 \\
0.5630\end{array}$ & $\begin{array}{r}0.3382 \\
-1.2369 \\
-1.0614 \\
-1.5201 \\
-1.2461 \\
-0.9692 \\
-0.7164 \\
-0.4873 \\
-0.2760 \\
-0.0756\end{array}$ \\
\hline
\end{tabular}

$\frac{\alpha}{\beta}=0.03$

$\mathrm{Y} / \Delta$

$0.0041^{-7.5206}$

$0.0085-3.6587$

$0.0131-2.3249$

$0.0183-1.6215$

$0.0241-1.1683$

$0.0307-0.8381$

$0.0386-0.5754$

$0.0482-0.3519$

$0.0602-0.1507$

$0.0756 \quad 0.0393$

$\begin{array}{lll}0.0962 & 0.2269\end{array}$

$0.1245 \quad 0.4201$

$0.1649 \quad 0.6273$

0.22530 .8588

$0.3200 \quad 1.1277$

$0.4781 \quad 1.4519$

$0.7611 \quad 1.8503$

$\begin{array}{ll}1.3082 & 2.3164\end{array}$

$2.4020 \quad 2.6627$

$4.1369 \quad 2.0120$

$\begin{array}{ll}4.7075 & -0.2997\end{array}$

$3.3293-1.7242$

$2.0919-1.7993$

$1.3914-1.5038$

$1.0036-1.1803$

$0.7775-0.8925$

$0.6396 .-0.6421$

$0.5539-0.4200$

$0.5025-0.2169$

$0.4761-0.0250$

$0.4709 \quad 0.1623$

$0.4866 \quad 0.3509$

0.52630 .5460

0.59720 .7528

0.71290 .9750

$0.8971 \quad 1.2119$

$1.1889 \quad 1.4489$
$1.6440 \quad 1.6321$

$2.2976 \quad 1.6184$

$3.0131 \quad 1.1685$

$\begin{array}{ll}3.3397 & 0.2537\end{array}$

$3.0115-0.6120$

$2.3870-1.0227$

$1.8305-1.0720$

$1.4308-0.9550$

$1.1608-0.7809$

$0.9810-0.5939$

$0.8628-0.4091$

$0.7881-0.2299$

$0.7464-0.0556$ $\frac{\alpha}{\beta}=0.05$

$0.0068^{Y / \Delta}-7.5207$

$0.0139-3.6589$

$0.0214-2.3253$

$0.0296-1.6221$

$0.0386-1.1692$

$0.0488-0.8392$

$0.0606-0.5770$

$0.0748-0.3541$

$0.0921-0.1539$

$0.1139 \quad 0.0347$

$0.1422 \quad 0.220$

$0.1804 \quad 0.4099$

$0.2336 \quad 0.6115$

$0.3108 \quad 0.8332$

$0.4284 \quad 1.0845$

$0.6167 \quad 1.3748$

$0.9351 \quad 1.7039$

\begin{tabular}{ll}
$1.4950 \quad 2.025$ \\
\hline
\end{tabular}

$2.4446 \quad 2.1152$

$3.6118 \quad 1.4047$

$3.8984-0.1729$

$3.0334-1.2433$

$2.1019-1.4479$

$1.4866-1.2916$

$1.1141-1.0522$

$0.8868-0.8132$

$\begin{array}{lll}0.7455 & -0.5927\end{array}$

$0.6582-0.3905$

$0.6077-0.2025$

$0.5850-0.0236$

$0.5863 \quad 0.1507$

0.66320 .4994

$0.8790 \quad 0.8586$

$\begin{array}{ll}0.0715 \quad 1.0317 \\ 1.3475 & 1.1732\end{array}$

$1.3475 \quad 1.1732$

$\begin{array}{ll}1.7225 & 1.2303\end{array}$

$2.1705 \quad 1.1143$

2.56790 .7461

$2.7168 \quad 0.1851$

$2.5427-0.3395$

$2.1843-0.6509$

$1.8125-0.7496$

$1.5070-0.7141$

$1.2798-0.6108$

$1.1189-0.4780$

$1.0096-0.3342$

$0.9405-0.1376$

$0.9040-0.0414$ $\frac{\alpha}{\beta}=0.07$

$\mathrm{Y} / . \Delta$

$0.0095-7.5209$

$0.0194-3.6593$

$0.0297-2.3258$

$0.0409-1.6229$

$0.0531-1.1703$

$\begin{array}{lll}0.0668 & -0.8408\end{array}$

$0.0826-0.5792$

$0.1013-0.3572$

$0.1238-0.1582$

$0.1518 \quad 0.0287$

$0.1877 \quad 0.2115$

0.23520 .3971

0.39280 .5919

0.39280 .8023

$\begin{array}{ll}0.5296 & 1.0338 \\ 0.7405 & 1.2878\end{array}$

$1.0771 \quad 1.5490$

$\begin{array}{ll}1.6171 & 1.7480\end{array}$

$2.4077 \quad 1.6792$

$3.2029 \quad 1.0128$

$3.3449-0.1219$

$2.7606-0.9319$

$2.0591-1.1711$

$1.5375-1.1033$

$1.1957-0.9305$

$0.9773-0.7352$

$0.8386-0.5436$

$0.7528-0.3620$

$0.7047-0.1900$

$0.6860-0.0255$

$0.6929 \quad 0.1342$

$0.7250 \quad 0.2907$

$0.7848 \quad 0.4449$

$0.8776 \quad 0.5953$

1.01120 .7365

$\begin{array}{ll}1.1952 & 0.8562\end{array}$

$\begin{array}{ll}1.4365 & 0.9302\end{array}$

$1.7285 \quad 0.9202$

2.03220 .7817

$2.2652 \quad 0.4992$

$\begin{array}{ll}2.3382 & 0.1304\end{array}$

$2.2308-0.2100$

$2.0087-0.4353$

$1.7580-0.5324$

$1.5328-0.5325$

$1.3525-0.4726$

$1.2181-0.3796$

$1.1243-0.2701$

$1.0648-0.1532$

$1.0351-0.0341$ 
TABLE V

Normalized Admittance $Y / \Delta$ of Loop Antennas in Dissipaitive Media

$\beta \mathrm{b}$

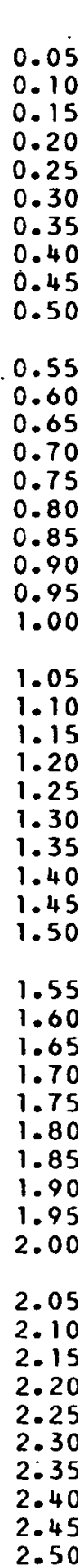

$$
\frac{\alpha}{\beta}=0.10
$$

$\mathrm{Y} / \Delta$

0.0136
0.0275

$0.0275-3.0600$

$0.0422-2.3270$

$0.0578-1.6246$

$0.0749-1.1727$

$0.0939-0.8441$

$0.1155-0.5836$

$0.1408-0.3632$

$0.1709-0.1665$

0.2080

0.0171

$0.2547 \quad 0.1950$

$0.3153 \quad 0.3732$

$0.3964 \quad 0.5562$

0.50820 .7474

$0.6670 \quad 0.9467$

$0.8980 \quad 1.1458$

$1.2365 \quad 1.3150$

$\begin{array}{ll}1.7134 & 1.3777\end{array}$

2.29121 .1906

2.7504

0.6387

$2.7882-0.1033$

2.4265

1.9561

1.5609

$-0.6519$

$-0.8737$

$1.0821-0.6282$

$0.9550-0.4755$

$0.8763-0.3237$

$0.8341-0.1764$

0.8213

0.8347

0.8733

0.9381

1.0315

1. 1559

1.3119

1.4943

1.6870

1.8595

1.9729

0.1019

0.2323

0.3550

0.4662

0.5586

0.6208

0.6363

0.5876

0.4646

0.2780

1.9987

0.0641

$\begin{array}{ll}.9374 & -0.1293\end{array}$

$1.8164-0.2673$

$.6716-0.3397$

$1.5312-0.3549$

$\begin{array}{ll}1.4106 & -0.3277\end{array}$

$1.3159-0.2728$

$\begin{array}{ll}1.2478 & -0.2012\end{array}$

$\begin{array}{lll}1.2051 & -0.1207\end{array}$

$1.1857-0.0369$
$\Omega=17$

\begin{tabular}{|c|c|c|c|c|c|c|c|}
\hline \multicolumn{2}{|c|}{$\begin{array}{c}\frac{\alpha}{\beta}=0.30 \\
Y / \Delta\end{array}$} & \multicolumn{2}{|c|}{$\frac{\alpha}{\beta}=0.50$} & \multicolumn{2}{|c|}{$\frac{\alpha}{\beta}=0.70$} & \multicolumn{2}{|c|}{$\frac{\alpha}{\beta}=1.00$} \\
\hline $\begin{array}{l}0.0405 \\
0.0819 \\
0.1247 \\
0.1697 \\
0.2179 \\
0.2703 \\
0.3281 \\
0.3927 \\
0.4660 \\
0.5498\end{array}$ & $\begin{array}{l}-7.5267 \\
-3.6714 \\
-2.3449 \\
-1.6502 \\
-1.2077 \\
-0.8909 \\
-0.6458 \\
-0.4455 \\
-0.2758 \\
-0.1291\end{array}$ & $\begin{array}{l}0.0675 \\
0.1359 \\
0.2061 \\
0.2791 \\
0.3557 \\
0.4368 \\
0.5232 \\
0.6153 \\
0.7133 \\
0.8167\end{array}$ & $\begin{array}{l}-7.5376 \\
-3.6938 \\
-2.3801 \\
-1.7001 \\
-1.2750 \\
-0.9792 \\
-0.7599 \\
-0.5915 \\
-0.4611 \\
-0.3622\end{array}$ & $\begin{array}{l}0.0943 \\
0.1894 \\
0.2860 \\
0.3847 \\
0.4859 \\
0.5894 \\
0.6948 \\
0.8010 \\
0.9062 \\
1.0076\end{array}$ & $\begin{array}{l}-7.5539 \\
-3.7272 \\
-2.4322 \\
-1.7733 \\
-1.3724 \\
-1.1046 \\
-0.9175 \\
-0.7858 \\
-0.6957 \\
-0.6393\end{array}$ & $\begin{array}{l}0.1345 \\
0.2686 \\
0.4019 \\
0.5338 \\
0.6629 \\
0.7871 \\
0.9041 \\
1.0111 \\
1.1057 \\
1.1860\end{array}$ & $\begin{array}{l}-7.5885 \\
-3.7977 \\
-2.5412 \\
-1.9242 \\
-1.5691 \\
-1.3507 \\
-1.2153 \\
-1.1355 \\
-1.0943 \\
-1.0803\end{array}$ \\
\hline $\begin{array}{l}0.6464 \\
0.7577 \\
0.8850 \\
1.0278 \\
1.1819 \\
1.3378 \\
1.4801 \\
1.5900 \\
1.6524 \\
1.6628\end{array}$ & $\begin{array}{r}-0.0019 \\
0.1060 \\
0.1918 \\
0.2501 \\
0.2736 \\
0.2548 \\
0.1906 \\
0.0862 \\
-0.0421 \\
-0.1712\end{array}$ & $\begin{array}{l}0.9240 \\
1.0325 \\
1.1380 \\
1.2354 \\
1.3192 \\
1.3848 \\
1.4297 \\
1.4545 \\
1.4622 \\
1.4576\end{array}$ & $\begin{array}{l}-0.2916 \\
-0.2481 \\
-0.2308 \\
-0.2380 \\
-0.2661 \\
-0.3098 \\
-0.3618 \\
-0.4148 \\
-0.4627 \\
-0.5015\end{array}$ & $\begin{array}{l}1.1022 \\
1.1867 \\
1.2585 \\
1.3157 \\
1.3582 \\
1.3871 \\
1.4047 \\
1.4140 \\
1.4178 \\
1.4186\end{array}$ & $\begin{array}{l}-0.6106 \\
-0.6045 \\
-0.6159 \\
-0.6392 \\
-0.6692 \\
-0.7009 \\
-0.7309 \\
-0.7569 \\
-0.7778 \\
-0.7936\end{array}$ & $\begin{array}{l}1.2513 \\
1.3021 \\
1.3399 \\
1.3670 \\
1.3859 \\
1.3990 \\
1.4082 \\
1.4152 \\
1.4209 \\
1.4260\end{array}$ & $\begin{array}{l}-1.0843 \\
-1.0993 \\
-1.1197 \\
-1.1415 \\
-1.1624 \\
-1.1810 \\
-1.1969 \\
-1.2103 \\
-1.2216 \\
-1.2312\end{array}$ \\
\hline $\begin{array}{l}1.6296 \\
1.5692 \\
1.4987 \\
1.4311 \\
1.3741 \\
1.3313 \\
1.3033 \\
1.2894 \\
1.2881 \\
1.2973\end{array}$ & $\begin{array}{l}-0.2800 \\
-0.3564 \\
-0.3982 \\
-0.4103 \\
-0.4000 \\
-0.3751 \\
-0.3419 \\
-0.3054 \\
-0.2693 \\
-0.2366\end{array}$ & $\begin{array}{l}1.4458 \\
1.4312 \\
1.4172 \\
1.4059 \\
1.3984 \\
1.3951 \\
1.3956 \\
1.3994 \\
1.4057 \\
1.4137\end{array}$ & $\begin{array}{l}-0.5296 \\
-0.5473 \\
-0.5562 \\
-0.5584 \\
-0.5562 \\
-0.5515 \\
-0.5459 \\
-0.5406 \\
-0.5365 \\
-0.5340\end{array}$ & $\begin{array}{l}1.4183 \\
1.4181 \\
1.4187 \\
1.4206 \\
1.4236 \\
1.4276 \\
1.4323 \\
1.4376 \\
1.4430 \\
1.4484\end{array}$ & $\begin{array}{l}-0.8050 \\
-0.8129 \\
-0.8183 \\
-0.8220 \\
-0.8250 \\
-0.8277 \\
-0.8305 \\
-0.8336 \\
-0.8372 \\
-0.8412\end{array}$ & $\begin{array}{l}1.4308 \\
1.4356 \\
1.4403 \\
1.4449 \\
1.4494 \\
1.4537 \\
1.4577 \\
1.4615 \\
1.4650 \\
1.4682\end{array}$ & $\begin{array}{l}-1.2396 \\
-1.2472 \\
-1.2543 \\
-1.2612 \\
-1.2678 \\
-1.2743 \\
-1.2808 \\
-1.2872 \\
-1.2935 \\
-1.2997\end{array}$ \\
\hline $\begin{array}{l}1.3150 \\
1.3388 \\
1.3664 \\
1.3954 \\
1.4236 \\
1.4489 \\
1.4699 \\
1.4855 \\
1.4956 \\
1.5005\end{array}$ & $\begin{array}{l}-0.2092 \\
-0.1886 \\
-0.1756 \\
-0.1705 \\
-0.1728 \\
-0.1815 \\
-0.1951 \\
-0.2119 \\
-0.2299 \\
-0.2474\end{array}$ & $\begin{array}{l}1.4227 \\
1.4319 \\
1.4409 \\
1.4493 \\
1.4567 \\
1.4632 \\
1.4687 \\
1.4733 \\
1.4772 \\
1.4805\end{array}$ & $\begin{array}{l}-0.5334 \\
-0.5345 \\
-0.5372 \\
-0.54 ! 1 \\
-0.5460 \\
-0.5515 \\
-0.5572 \\
-0.5628 \\
-0.5683 \\
-0.5735\end{array}$ & $\begin{array}{l}1.4537 \\
1.4586 \\
1.4632 \\
1.4675 \\
1.4714 \\
1.4749 \\
1.4782 \\
1.4812 \\
1.4839 \\
1.4865\end{array}$ & $\begin{array}{l}-0.8455 \\
-0.8502 \\
-0.8551 \\
-0.8601 \\
-0.8651 \\
-0.8701 \\
-0.8751 \\
-0.8800 \\
-0.8848 \\
-0.8894\end{array}$ & $\begin{array}{l}1.4712 \\
1.4738 \\
1.4762 \\
1.4784 \\
1.4803 \\
1.4820 \\
1.4836 \\
1.4849 \\
1.4861 \\
1.4871\end{array}$ & $\begin{array}{l}-1.3059 \\
-1.3119 \\
-1.3179 \\
-1.3237 \\
-1.3294 \\
-1.3350 \\
-1.3405 \\
-1.3459 \\
-1.3512 \\
-1.3564\end{array}$ \\
\hline $\begin{array}{l}1.5011 \\
1.4985 \\
1.4941 \\
1.4890 \\
1.4843 \\
1.4807 \\
1.4788 \\
1.4788 \\
1.4806 \\
1.4841\end{array}$ & $\begin{array}{l}-0.2629 \\
-0.2754 \\
-0.2847 \\
-0.2906 \\
-0.2935 \\
-0.2940 \\
-0.2928 \\
-0.2906 \\
-0.2881 \\
-0.2858\end{array}$ & $\begin{array}{l}1.4834 \\
1.4860 \\
1.4885 \\
1.4910 \\
1.4934 \\
1.4959 \\
1.4985 \\
1.5011 \\
1.5037 \\
1.5064\end{array}$ & $\begin{array}{l}-0.5783 \\
-0.5827 \\
-0.5868 \\
-0.5906 \\
-0.5942 \\
-0.5977 \\
-0.6011 \\
-0.6045 \\
-0.6080 \\
-0.6115\end{array}$ & $\begin{array}{l}1.4889 \\
1.4912 \\
1.4934 \\
1.4954 \\
1.4973 \\
1.4991 \\
1.5009 \\
1.5025 \\
1.5040 \\
1.5054\end{array}$ & $\begin{array}{l}-0.8940 \\
-0.8986 \\
-0.9030 \\
-0.9074 \\
-0.9118 \\
-0.9161 \\
-0.9204 \\
-0.9247 \\
-0.9289 \\
-0.9331\end{array}$ & $\begin{array}{l}1.4879 \\
1.4886 \\
1.4891 \\
1.4895 \\
1.4897 \\
1.4898 \\
1.4898 \\
1.4897 \\
1.4895 \\
1.4891\end{array}$ & $\begin{array}{l}-1.3615 \\
-1.3665 \\
-1.3715 \\
-1.3763 \\
-1.3811 \\
-1.3858 \\
-1.3904 \\
-1.3950 \\
-1.3994 \\
-1.4039\end{array}$ \\
\hline
\end{tabular}


TABLE VI

Normalized Admittance $\mathrm{Y} / \Delta$ of Loop Antennas in Dissipative Media

$\beta b \quad \frac{\alpha}{\beta}=0.00$

\begin{tabular}{lll} 
& \multicolumn{2}{c}{$\mathrm{Y} / \Delta$} \\
0.05 & 0.0001 & -6.3809 \\
0.10 & 0.0002 & -3.1057 \\
0.15 & 0.0005 & -1.9752 \\
0.20 & 0.0009 & -1.3794 \\
0.25 & 0.0016 & -0.9958 \\
0.30 & 0.0026 & -0.7164 \\
0.35 & 0.0039 & -0.4942 \\
0.40 & 0.0059 & -0.3050 \\
0.45 & 0.0087 & -0.1344 \\
0.50 & 0.0128 & 0.0272
\end{tabular}

0.50

0.55

0.60

0.65

0.70
0.75

0.80

0.85

0.90

0.95

1.00

1.05
1.10

1.05
1.15

1.20

1.25

1.30

1.35
1.40

1.45

1.55
1.60

1.60

1.65
1.75

1.80

1.85

1.90

1.95
2.00

2.05

2.10

2.15
2.20

2.25

2.30

2.35

2.40

2.45

2.50
$\Omega=20$

\begin{tabular}{|c|c|c|c|c|c|c|c|}
\hline \multicolumn{2}{|c|}{$\frac{\alpha}{\beta}=0.01$} & \multicolumn{2}{|c|}{$\frac{\alpha}{\beta}=0.03$} & \multicolumn{2}{|c|}{$\frac{\alpha}{\beta}=0.05$} & \multicolumn{2}{|c|}{$\frac{\alpha}{\beta}=0.07$} \\
\hline $\begin{array}{l}0.0012 \\
0.0025 \\
0.0040 \\
0.0057 \\
0.0077 \\
0.0101 \\
0.0132 \\
0.0170 \\
0.0222 \\
0.0291\end{array}$ & $\begin{array}{l}Y / \Delta \\
-6.3810 \\
-3.1058 \\
-1.9752 \\
-1.3794 \\
-0.9959 \\
-0.7165 \\
-0.4944 \\
-0.3053 \\
-0.1349 \\
0.0264\end{array}$ & $\begin{array}{l}0.0034 \\
0.0070 \\
0.0109 \\
0.0151 \\
0.0198 \\
0.0252 \\
0.0316 \\
0.0394 \\
0.0490 \\
0.0614\end{array}$ & $\begin{array}{l}/ \Delta \\
-6.3310 \\
-3.1359 \\
-1.9754 \\
-1.3797 \\
-0.9763 \\
-0.7171 \\
-0.4752 \\
-0.3765 \\
-0.1366 \\
0.0239\end{array}$ & $\begin{array}{l}0.0057 \\
0.0116 \\
0.0178 \\
0.0245 \\
0.0319 \\
0.0403 \\
0.0501 \\
0.0616 \\
0.0758 \\
0.0936\end{array}$ & $\begin{array}{l}/ \Delta \\
-6.3811 \\
-3.1061 \\
-1.9757 \\
-1.3802 \\
-0.9970 \\
-0.7180 \\
-0.4965 \\
-0.3083 \\
-0.1392 \\
0.0202\end{array}$ & $\begin{array}{l} \\
0.0079 \\
0.0161 \\
0.0247 \\
0.0339 \\
0.0441 \\
0.0554 \\
0.0685 \\
0.0838 \\
0.1024 \\
0.1255\end{array}$ & $\begin{array}{l}\Delta \\
-0.3812 \\
-3.1064 \\
-1.9762 \\
-1.3809 \\
-0.9979 \\
-0.7193 \\
-0.4983 \\
-0.3108 \\
-0.1427 \\
0.0153\end{array}$ \\
\hline $\begin{array}{l}0.0387 \\
0.0525 \\
0.0731 \\
0.1054 \\
0.1585 \\
0.2527 \\
0.4367 \\
0.8473 \\
1.9280 \\
4.7106\end{array}$ & $\begin{array}{l}0.1865 \\
0.3526 \\
0.5329 \\
0.7381 \\
0.9838 \\
1.2956 \\
1.7162 \\
2.3137 \\
3.0949 \\
2.8443\end{array}$ & $\begin{array}{l}0.0779 \\
0.1005 \\
0.1329 \\
0.1814 \\
0.2581 \\
0.3879 \\
0.6265 \\
1.1092 \\
2.1525 \\
3.9455\end{array}$ & $\begin{array}{l}0.1828 \\
0.3468 \\
0.5236 \\
0.7226 \\
0.9566 \\
1.2440 \\
1.6085 \\
2.0598 \\
2.4405 \\
1.7582\end{array}$ & $\begin{array}{l}0.1168 \\
0.1479 \\
0.1914 \\
0.2550 \\
0.3526 \\
0.5113 \\
0.7868 \\
1.2922 \\
2.2026 \\
3.3597\end{array}$ & $\begin{array}{l}0.1774 \\
0.3386 \\
0.5109 \\
0.7019 \\
0.9212 \\
1.1796 \\
1.4822 \\
1.7947 \\
1.9014 \\
1.1603\end{array}$ & $\begin{array}{l}0.1552 \\
0.1944 \\
0.2484 \\
0.3256 \\
0.4409 \\
0.6216 \\
0.9175 \\
1.4103 \\
2.1640 \\
2.9227\end{array}$ & $\begin{array}{l}0.1703 \\
0.3282 \\
0.4949 \\
0.6764 \\
0.8789 \\
1.1055 \\
1.3460 \\
1.5394 \\
1.4783 \\
0.7969\end{array}$ \\
\hline $\begin{array}{l}5.485 .3 \\
2.7570 \\
1.4322 \\
0.8759 \\
0.6054 \\
0.4565 \\
0.3676 \\
0.3122 \\
0.2778 \\
0.2584\end{array}$ & $\begin{array}{l}-1.3368 \\
-2.5378 \\
-2.0315 \\
-1.5196 \\
-1.1327 \\
-0.8356 \\
-0.5956 \\
-0.3912 \\
-0.2083 \\
-0.0370\end{array}$ & $\begin{array}{l}4.2674 \\
2.6900 \\
1.5893 \\
1.0309 \\
0.7367 \\
0.5696 \\
0.4695 \\
0.4086 \\
0.3730 \\
0.3563\end{array}$ & $\begin{array}{l}-0.7195 \\
-1.8264 \\
-1.6909 \\
-1.3508 \\
-1.0402 \\
-0.7810 \\
-0.5022 \\
-0.3711 \\
-0.1979 \\
-0.0347\end{array}$ & $\begin{array}{l}3.4877 \\
2.5089 \\
1.6491 \\
1.1363 \\
0.8419 \\
0.6677 \\
0.5619 \\
0.4981 \\
0.4627 \\
0.4489\end{array}$ & $\begin{array}{l}-0.4540 \\
-1.3393 \\
-1.3889 \\
-1.1811 \\
-0.9415 \\
-0.7213 \\
-0.5257 \\
-0.3500 \\
-0.1883 \\
-0.0353\end{array}$ & $\begin{array}{l}2.9593 \\
2.3089 \\
1.6490 \\
1.2011 \\
0.9227 \\
0.7506 \\
0.6441 \\
0.5801 \\
0.5460 \\
0.5351\end{array}$ & $\begin{array}{l}-0.3265 \\
-1.0138 \\
-1.1405 \\
-1.0242 \\
-0.8442 \\
-0.0605 \\
-0.4884 \\
-0.3291 \\
-0.1802 \\
-0.0386\end{array}$ \\
\hline $\begin{array}{l}0.2514 \\
0.2566 \\
0.2763 \\
0.3158 \\
0.3862 \\
0.5103 \\
0.7369 \\
1.1755 \\
2.0572 \\
3.5455\end{array}$ & $\begin{array}{l}0.1305 \\
0.3012 \\
0.4824 \\
0.6826 \\
0.9131 \\
1.1883 \\
1.5247 \\
1.9215 \\
2.2496 \\
1.8339\end{array}$ & $\begin{array}{l}0.3556 \\
0.3712 \\
0.4060 \\
0.4666 \\
0.5655 \\
0.7255 \\
0.9868 \\
1.4131 \\
2.0605 \\
2.7850\end{array}$ & $\begin{array}{l}0.1246 \\
0.2855 \\
0.4533 \\
0.8333 \\
0.8502 \\
1.0461 \\
1.2714 \\
1.4580 \\
1.4539 \\
0.9691\end{array}$ & $\begin{array}{l}0.4539 \\
0.4779 \\
0.5242 \\
0.5991 \\
0.7137 \\
0.3853 \\
1.1376 \\
1.4911 \\
1.9246 \\
2.3015\end{array}$ & $\begin{array}{l}0.1136 \\
0.2622 \\
0.4136 \\
0.5697 \\
0.7299 \\
0.8871 \\
1.0198 \\
1.0752 \\
0.9568 \\
0.5732\end{array}$ & $\begin{array}{l}0.5449 \\
0.5754 \\
0.6293 \\
0.7119 \\
0.8311 \\
0.9972 \\
1.2189 \\
1.4921 \\
1.7776 \\
1.9871\end{array}$ & $\begin{array}{l}0.0984 \\
0.2331 \\
0.3664 \\
0.4977 \\
0.6225 \\
0.7298 \\
0.7905 \\
0.7832 \\
0.6421 \\
0.3566\end{array}$ \\
\hline $\begin{array}{l}4.2701 \\
3.0998 \\
1.9261 \\
1.2608 \\
0.8977 \\
0.6881 \\
0.5603 \\
0.4799 \\
0.4296 \\
0.4006\end{array}$ & $\begin{array}{l}-0.1374 \\
-1.5752 \\
-1.6954 \\
-1.4267 \\
-1.1272 \\
-0.8629 \\
-0.6353 \\
-0.4352 \\
-0.2534 \\
-0.0924\end{array}$ & $\begin{array}{l}3.0304 \\
2.5661 \\
1.9127 \\
1.4092 \\
1.0770 \\
0.8640 \\
0.7270 \\
0.6394 \\
0.5858 \\
0.5578\end{array}$ & $\begin{array}{l}-0.0134 \\
-0.8202 \\
-1.0859 \\
-1.0537 \\
-0.8998 \\
-0.3211 \\
-0.5454 \\
-0.3792 \\
-0.2217 \\
-0.0705\end{array}$ & $\begin{array}{l}2.3988 \\
2.1653 \\
1.7883 \\
1.4391 \\
1.1726 \\
0.8841 \\
0.8555 \\
0.7710 \\
0.7196 \\
0.6947\end{array}$ & $\begin{array}{r}0.0037 \\
-0.4822 \\
-0.7240 \\
-0.7647 \\
-0.6975 \\
-0.5843 \\
-0.4551 \\
-0.3226 \\
-0.1915 \\
-0.0627\end{array}$ & $\begin{array}{l}2.0269 \\
1.8894 \\
1.0572 \\
1.4179 \\
1.2160 \\
1.0616 \\
0.9506 \\
0.8757 \\
0.8302 \\
0.8098\end{array}$ & $\begin{array}{l}-0.0050 \\
-0.3170 \\
-0.5011 \\
-0.5612 \\
-0.5378 \\
-0.4678 \\
-0.3749 \\
-0.2722 \\
-0.1661 \\
-0.0600\end{array}$ \\
\hline
\end{tabular}


TABLE VI

Normalized Admittance $\mathrm{Y} / \triangle$ of Loop Antennas in Dissipative Media

$$
\frac{\alpha}{\beta}=0.10
$$

$0.0113-6.3815$

$0.0229-3.1070$

$0.0351-1.9772$

$0.0480-1.3823$

$0.0622-0.9999$

$0.0959-0.5020$

$0.1169-0.3157$

$0.1420-0.1495$

$0.1728 \quad 0.0058$

0.45

0.55

0.6

0.65

0.70

0.75

0.75

0.80

0.85
0.90

0.90

1.00

1.05

1.10

1.15
1.20

1.25

1.30

1.35

1.40

1.45

1.55

1.60

1.65

1.70

1.75

1.80
1.85

1.90

1.95

2.00

2.05

2.10

2.15

2.20

2.25

2.30

2.35

2.40

2.45
2.50

$0.2117 \quad 0.1567$

$0.2624 \quad 0.3084$

$\begin{array}{ll}0.3305 & 0.4651 \\ 0.4251 & 0.6303\end{array}$

$0.4251 \quad 0.6303$

$0.5609 \quad 0.8048$

$1.0632 \quad 1.1375$

$1.5000 \quad 1.1974$

$\begin{array}{ll}2.0413 & 1.0150 \\ 2.4573 & 0.4673\end{array}$

$2.4343-0.2422$

$2.0421-0.7142$

$1.5945-0.8641$

$1.2458-0.8260$

$1.0062-0.7116$

$0.8488-0.5744$

$0.7483-0.4351$

$0.6881-0.3003$

$0.6577-0.1716$

$0.6514-0.0487$

$0.6666 \quad 0.0690$

$\begin{array}{ll}0.7030 & 0.1815 \\ 0.7619 & 0.2876\end{array}$

$0.7619 \quad 0.2876$

$\begin{array}{ll}0.9578 & 0.4645 \\ 1.0993 & 0.5178\end{array}$

$1.2660 \quad 0.5280$

$1.4425 \quad 0.4769$

$\begin{array}{ll}1.5982 & 0.3543\end{array}$

1.69330 .1719

$1.7011-0.0310$

$1.6264-0.2059$

$1.5014-0.3217$

$1.3621-0.3741$

$1.2334-0.3749$

$1.1271-0.3404$

$1.0463-0.2841$

$\begin{array}{ll}0.9902 & -0.2157\end{array}$

$\begin{array}{ll}0.9565 & -0.1415 \\ 0.9433 & -0.0659\end{array}$
$0.0113^{\mathrm{Y} / \Delta}$

$0.0780-0.7220$

$\begin{array}{ll}0.8458 & 0.3841 \\ 0.0578 & 0.4645\end{array}$ $\frac{\alpha}{\beta}=0.30$

$\mathrm{Y} / \Delta$

$\frac{\alpha}{\beta}=0.50$

$\mathrm{Y} / \Delta$

$0.0338^{-6}-3861$

$0.0682-3.1164$

$0.1038-1.9920$

$0.1414-1.4036$

$0.1816-1.0290$

$0.2254-0.7611$

$0.2738-0.5538$

$0.3281-0.3845$

$0.3897-0.2410$

$0.4606-0.1169$

$0.5425-0.0093$

$0.6375 \quad 0.0820$

$0.7467 \quad 0.1545$

$0.8698 \quad 0.2031$

$1.0034 \quad 0.2209$

$\begin{array}{ll}1.1388 & 0.2009\end{array}$

$1.2616 \quad 0.1399$

$1.3544 \quad 0.0430$

$\begin{array}{ll}1.4031 & -0.0744 \\ 1.4043 & -0.1905\end{array}$

$1.3669-0.2858$

$1.3069-0.3501$

$1.2401-0.3827$

$1.1779-0.3889$

$1.0889-0.3513$

$\begin{array}{lll}1.0648 & -0.3201\end{array}$

$1.0532-0.2868$

$1.0526-0.2546$

$1.0613-0.2258$

$1.0771-0.2022$

$1.0981-0.1848$

$1.1222-0.1744$

$1.1472-0.1710$

$1.1711-0.1742$

$1.1921-0.1831$

$1.2090-0.1963$

$1.2210-0.2120$

$1.2279-0.2284$

$1.2301-0.2441$

$1.2286-0.2577$

$1.2244-0.2686$

$1.2188-0.2762$

$1.2128-0.2808$

$1.2074-0.2827$

$1.2033-0.2325$

$1.2007-0.2309$

$1.1999-0.2784$

$1.2008-0.2758$

$1.2033-0.2736$
1.2129
$1.1267-0.3761$
$\Omega=20$

\begin{tabular}{|c|c|c|c|c|c|}
\hline \multicolumn{2}{|c|}{$\mathrm{Y} / \Delta$} & \multicolumn{2}{|c|}{$\mathrm{Y} / \Delta$} & \multicolumn{2}{|c|}{$\mathrm{Y} / \Delta$} \\
\hline $\begin{array}{l}0.0562 \\
0.1132 \\
0.1717 \\
0.2326 \\
0.2966 \\
0.3645 \\
0.4369 \\
0.5143 \\
0.5968 \\
0.6842\end{array}$ & $\begin{array}{l}-6.3952 \\
-3.1351 \\
-2.0214 \\
-1.4452 \\
-1.0852 \\
-0.8349 \\
-0.6495 \\
-0.5072 \\
-0.3972 \\
-0.3141\end{array}$ & $\begin{array}{l}0.0786 \\
0.1578 \\
0.2383 \\
0.3207 \\
0.4051 \\
0.4917 \\
0.5800 \\
0.6690 \\
0.7571 \\
0.8421\end{array}$ & $\begin{array}{l}-0.4087 \\
-3.1629 \\
-2.0648 \\
-1.5062 \\
-1.1666 \\
-0.9399 \\
-0.7817 \\
-0.6706 \\
-0.5950 \\
-0.5481 \\
-. . .\end{array}$ & $\begin{array}{l}0.1120 \\
0.2237 \\
0.3349 \\
0.4448 \\
0.5524 \\
0.6560 \\
0.7533 \\
0.8421 \\
0.9202 \\
0.9859\end{array}$ & $\begin{array}{l}-6.437 \\
-3.221 \\
-2.155 \\
-1.632 \\
-1.331 \\
-1.145 \\
-1.031 \\
-0.963 \\
-0.929 \\
-0.918\end{array}$ \\
\hline $\begin{array}{l}0.7750 \\
0.8669 \\
0.9563 \\
1.0384 \\
1.1084 \\
1.1621 \\
1.1974 \\
1.2149 \\
1.2174 \\
1.2096\end{array}$ & $\begin{array}{l}-0.2552 \\
-0.2197 \\
-0.2069 \\
-0.2155 \\
-0.2423 \\
-0.2824 \\
-0.3293 \\
-0.3765 \\
-0.4185 \\
-0.4518\end{array}$ & $\begin{array}{l}0.9211 \\
0.9914 \\
1.0504 \\
1.0967 \\
1.1300 \\
1.1516 \\
1.1635 \\
1.1683 \\
1.1686 \\
1.1667\end{array}$ & $\begin{array}{l}-0.5250 \\
-0.5214 \\
-0.5326: 1 \\
-0.5540 \\
-0.5807: \\
-0.6086: 14 \\
-0.6345 \\
-0.6566 \\
-0.6739 \\
-0.6868 \\
\end{array}$ & $\begin{array}{l}1.0386 \\
1.0788 \\
1.1079 \\
1.1279 \\
1.1409 \\
1.1492 \\
1.1544 \\
1.1578 \\
1.1604 \\
1.1626 \\
1.1\end{array}$ & $\begin{array}{l}-0.922 \\
-0.935 \\
-0.952 \\
-0.970 \\
-0.987 \\
-1.002 \\
-1.015 \\
-1.025 \\
-1.034\end{array}$ \\
\hline $\begin{array}{l}1.1958 \\
1.1803 \\
1.1658 \\
1.1542 \\
1.1463 \\
1.1422 \\
1.1416 \\
1.1439 \\
1.1485 \\
1.1545\end{array}$ & $\begin{array}{l}-0.4752 \\
-0.4893 \\
-0.4956 \\
-0.4963 \\
-0.4932 \\
-0.4882 \\
-0.4826 \\
-0.4775 \\
-0.4736 \\
-0.4712\end{array}$ & $\begin{array}{l}1.1641 \\
1.1619 \\
1.1606 \\
1.1605 \\
1.1616 \\
1.1636 \\
1.1663 \\
1.1695 \\
1.1729 \\
1.1762\end{array}$ & $\begin{array}{l}-0.69 .56 t 6 \\
-0.70 .15^{2}: 4 \\
-0.70 .5234 \\
-0.7076 \\
-0.7093 \\
-0.7109 \\
-0.71 .27 \\
-0.714 .8 \\
-0.7174 \\
-0.7203\end{array}$ & $\begin{array}{l}1.1648 \\
1.1670 \\
1.1693 \\
1.1716 \\
1.1738 \\
1.1759 \\
1.1779 \\
1.1797 \\
1.1813 \\
1.1827\end{array}$ & $\begin{array}{l}-1.047 \\
-1.052 \\
-1.057 \\
-1.062 \\
-1.066 \\
-1.071 \\
-1.075 \\
-1.080 \\
-1.084 \\
-1.089\end{array}$ \\
\hline $\begin{array}{l}1.1613 \\
1.1683 \\
1.1749 \\
1.1810 \\
1.1863 \\
1.1906 \\
1.1941 \\
1.1969 \\
1.1990 \\
1.2006\end{array}$ & $\begin{array}{l}-0.4706 \\
-0.4715 \\
-0.4738 \\
-0.4772 \\
-0.4814 \\
-0.4861 \\
-0.4909 \\
-0.4956 \\
-0.5002 \\
-0.5044\end{array}$ & $\begin{array}{l}1.1795 \\
1.1825 \\
1.1852 \\
1.1876 \\
1.1897 \\
1.1916 \\
1.1932 \\
1.1946 \\
1.1959 \\
1.1970\end{array}$ & $\begin{array}{l}-0.7236 \\
-0.7272 \\
-0.7309 \\
-0.7347 \\
-0.7386 \\
-0.7424 \\
-0.7462 \\
-0.7499 \\
-0.7535 \\
-0.7571\end{array}$ & $\begin{array}{l}1.1839 \\
1.1849 \\
1.1857 \\
1.1863 \\
1.1868 \\
1.1871 \\
1.1873 \\
1.1873 \\
1.1873 \\
1.1871\end{array}$ & $\begin{array}{l}-1.0935 \\
-1.0977 \\
-1.1019 \\
-1.1060 \\
-1.1100 \\
-1.1139 \\
-1.1177 \\
-1.1215 \\
-1.1252 \\
-1.1288\end{array}$ \\
\hline $\begin{array}{l}1.2020 \\
1.2031 \\
1.2042 \\
1.2053 \\
1.2064 \\
1.2076 \\
1.2089 \\
1.2102 \\
1.2116 \\
1.2129\end{array}$ & $\begin{array}{l}-0.5083 \\
-0.5118 \\
-0.5150 \\
-0.5180 \\
-0.5208 \\
-0.5235 \\
-0.5261 \\
-0.5287 \\
-0.5314 \\
-0.5341\end{array}$ & $\begin{array}{l}1.1981 \\
1.1990 \\
1.1998 \\
1.2006 \\
1.2013 \\
1.2019 \\
1.2025 \\
1.2029 \\
1.2033 \\
1.2037\end{array}$ & $\begin{array}{l}-0.7605 \\
-0.7639 \\
-0.7672 \\
-0.7705 \\
-0.7738 \\
-0.7770 \\
-0.7802 \\
-0.7834 \\
-0.7865 \\
-0.7897\end{array}$ & $\begin{array}{l}1.1868 \\
1.1864 \\
1.1859 \\
1.1853 \\
1.1846 \\
1.1838 \\
1.1829 \\
1.1820 \\
1.1809 \\
1.1798\end{array}$ & $\begin{array}{l}-1.132 \\
-1.1358 \\
-1.1392 \\
-1.142 \\
-1.145 \\
-1.149 \\
-1.152 \\
-1.1554 \\
-1.1585 \\
-1.1615\end{array}$ \\
\hline
\end{tabular}

$$
\frac{\alpha}{\beta}=1.00
$$

$$
\frac{\alpha}{\beta}=0.70
$$$$
\mathrm{Y} / \triangle
$$

$0.0786-0.4087$

$0.1578-3.162$

$0.2383-2.064$

$0.4051-1.1666$

$-0.9399$

$-0.781$

0.6706

$\begin{array}{ll}1.1809 & -1.1585 \\ 1.1798 & -1.1615\end{array}$ 